LA-7683-MS

Informal Report

Special Distribution

Issued: February 1979

\title{
Design and Operation of the LAMPF Auxiliary Controller \\ High-Speed Remote Processing on the CAMAC Dataway
}

\author{
Donald R. Machen
}

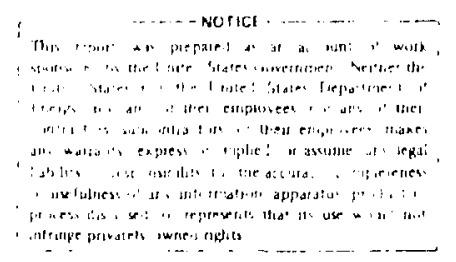


I. INTRODUCTION ................. 1

Reference Documents .......... 2

System Configuration ......... 2

ACC Configuration ........... 3

II. PROTOTYPE ACC BOARDS* ......... 6 63Y-182694, Shts 1,2

A. LST-11/2 Processor Board ....... 6 63Y-182704, Shts 1-5

B. LSI-11/2 Board Hardware . . . . . 8

1. Fixed PROM and Boot Memory ..... 8

2. Fixed RAM .......... . . 8

3. Memory Page Control ...... 8

4. Multifrequency Clock ...... . 9

5. Terminal Port ........ . 9

C. Dataway Access Port . . . . . . 11 63Y-182705, Shts 1-3

D. ACC Memory . . . . . . . . . 13 63Y-182708/710

E. Control Port.......... 17 63Y-182706, Sht' 1-5

F. The Special Processor Unit...... 23 63Y-182707, Shts 1-5

G. The SPU PROM Simulator....... 29 63Y-182711, Shts 1-3

H. Minimum Controller....... 31 $63 Y-182712$, Sht 1

III. ICC SOFTWARE ............ 32

A. The Basic Debug Code ........ . 33

B. SPU Microcode ......... 35 APPENDIX ........................ 44

CAMAC Auxiliary Controller Concepts . . . 45 CONVENTIDNAL CAMAC SYSTEMS AND THE ACC . . 52

*Prototype documentation available from the LAMPF Erawing Office under $63 \gamma-182693$, Sheets 1-28. 


\section{FIGURES}

\section{Page}

Fig. 1. System configuration ....... 3

Fig. 2. Black diagram of ACC and..... 4 address space allocation $(63 Y-182694 / 1)$

Fig. 3. Multicrate expansion ....... 5

Fig. 4. Special processor organization. . . 24

Fig. 5. Debugger commands. . . . . . . 33

Fig. 6. Sample ACBUG session . . . . . . 34

Fig. 7. The special processor unit. . . . 36 microcode format

Fig. 8. Interim coding sheet . . . . . . 43 


\title{
DESIGN AND OPERATION OF THE LAMPF AUXILIARY CONTROLLER
}

High-Speed Remote Processing on the CAMAC Dataway

by

Donald R. Machen

\begin{abstract}
A CAMAC Auxiliary Controller has been developed to further the concepts of distributed processing in both process control and experiment data-acquisition systems. The Auxiliary Controller is built around a commercially available 16-bit microcomputer and a highspeed bit-sliced microprocessor capable of instruction execution times of $140 \mathrm{~ns}$. The modular nature of the controller allows the user to tailor the controller capabilities to the system problem, while maintaining the interface techniques of the CAMAC Standard.
\end{abstract}

\section{INTRODUCTION}

This report covers both the design details and operation of an LSI-11/2* CAMAC Auxiliary Crate Controller (ACC). I will also attempt to convey how such a device can be used wi th CAMAC data and control systems and what options may be taker with respect, to the several modules that comprise the ACC.

The Auxiliary Controller discussed in this report is based upon a design done around the Ferranti FTOOL (a 16-bit single-chip microprocessor) that is presently operational at the Daresbury Laboratory in England. The concept has remained the same between the two ACCs; only the necessary hardware details have changed to accommodate the LSI-11/2 "Q-Bus."

In order to fully understand the contents of this report, the reader must have a reasonable grounding in the reference documents listed below. Without some knowledge of the CAMAC standard, or the material published by DEC and Advanced Micro Devices (AMD), the technical details of the ACC may become somewhat opaque.

*Digital Equipment Corporation (DEC) trademark 


\section{Reference Documents}

1. IEEE Standard 583-1975 (EUR-4100e), "Modular Instrumentation and Digital Interface System - CAMAC"

2. IEEE Standard 595-1976 (EUR-6100e), "Serial Highway Interface System - CAMAC"

3. IEEE Standard 596-1976 (EUR-4600e), "Parallel Highway Interface System CAMAC"

4. DOE/EV-0007 (EUR-6500e), "Multiple Controllers in a CAMAC Crate"

5. TID-26618, "CAMAC Tutorial Articles," USD0E Publication Covcring All Aspects of the CAMAC System

6. Digital Equipment Corporation, "1978 Microcomputer Handbook and Addenda Covering the LSI-11/2"

7. Advancer Micro Devices, Inc., AMD-2900, "Bipolar Microprocessor Family Data Book"

8. D. R. Machen, C. G. Ratcliffe, and A. C. Pealfield, "A Multi-Microprocessor Auxiliary Crate Controller for Front-End Data Processing in CAMAC," Daresbury Laboratory/Science Research Council, England, report DL/CSE/7?-1, and

IEEE Transactions on Nuclear Science, Vol. NS 25, No. 1, p 711, February 1978.

Reference documents 1 to 3 describe fully the CAMAC system of instrumentation, while Ref. 4 explains how multiple auxiliary controllers may share the Dataway of a single CAMAC crate. Reference 5 contains an interesting and informative set of papers on CAMAC. - It is recominended reading for anybody attempting to plow through the formal CAMAC specifications. References 6 and 7 are essential to the understanding of the ACC described in this report, while ref. 8 discusses the ACC based on the Ferranti microprocessor.

The reader, at this point, should turn to Appendix $A$ of this report in order to review the initial system design concepts upon which the LAMPF* ACC is based. An understanding of the ideas behind front-end and parallel processing in a CAMAC instrumentation environment will aid in understanding the details that follow in this report.

\section{Sys tem Configuration}

The CAMAC system configuration shown in Fig. 1 indicates where, in a large CAMAC system, multiple auxiliary controllers can be included. The motivating factor for auxiliary controllers is two-fold: Firstly, remote parallel processing can be accomplished in CAMAC crates interfaced to a larger hos $t$ computer, and secoind?y, high-speed, front-end processing, or filtering, can be realized in data collection systems. The first case is generally useful in distributed control systems, wherease the second case finds use in experiments (in various fields) where data rates are high and not all the data are considered "good." Further, the overall gains possible in system throughput and response to remote stimuli are also motivating factors. 


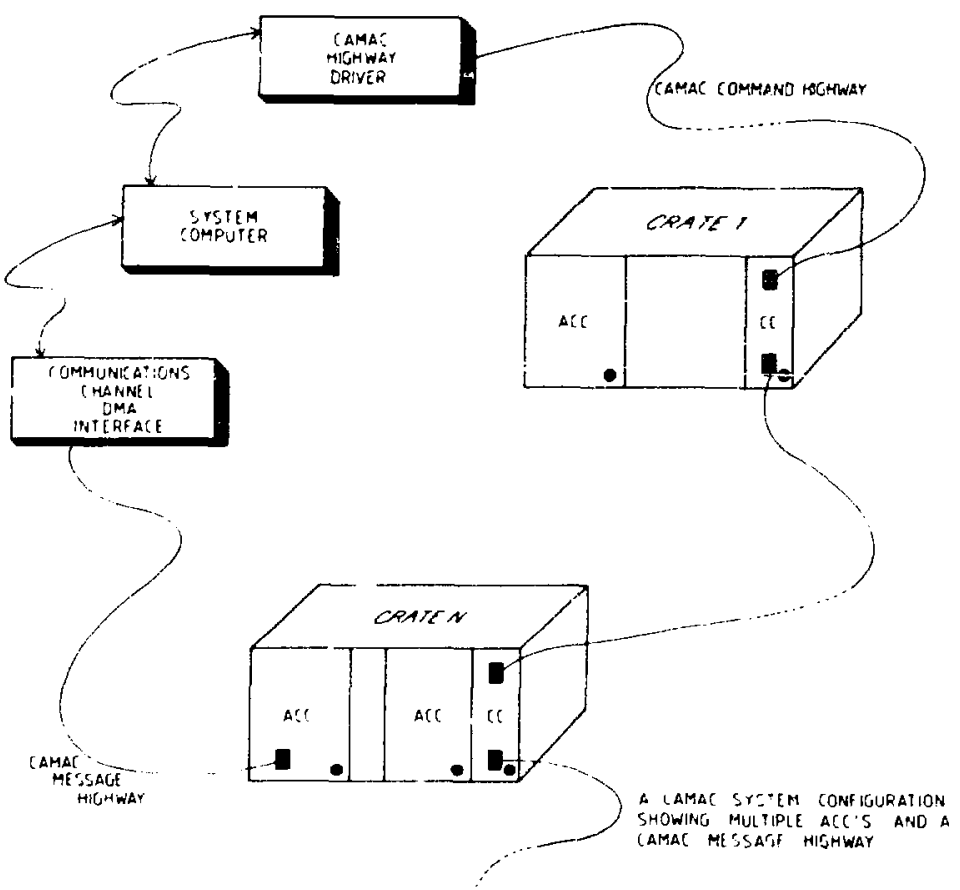

Fig. 1. System configuration.
Although rot physically provided with either the Daresbury or the LAMPF prototype versions of the Auxiliary Controller, a high-speed (2-MHz) bit-serial, messageoriented block-transfer channel can easily be added to complement the well-kriown single-action CAMAC highways. Such an autonomous message charnel can gireatly enhance the data transfer rate and throughput of a front-end processor ACC.

A practical limit to the number of ACCS per crate is two to three. Al though DOE/ EV-0007 (EUR-6500e) allows eight ACCS, one soon realizes that space and power constraints prevail. The spectre of a crate full of ACCs, all talking to one another and to the sys tem computer, may be an interesting exercise but has little value in the practical world of data acquisition and process control.

\section{ACC Configuration}

The LAMPF ACC is modular and flexible from the point of view that not all components are needed in order to configure a working system. Figure 2 shows the basic idea--that of a microprocessor-implemented controller, with Control Port, Dataway Access Port, processor memory, and a fast firmware subroutine facility. The Control Port provides the ACC access to all CAMAC Dataway signal lines with the exception of station number lines and LAM lines. The latter two are sent and received, respectively, via the Auxiliary Controller Bus (ACB). Further, priority arbitration for Dataway "mastership" takes place along the ACB (the reader is referred to DOE/EV-0007 for complete details on the ACB). The Dataway Access Port allows a system controller, via the CAMAC Command Highway, to read and write ACC memory during program load or execution times.

Several different memory configurations are possible, depending upon the nature of the ACC application. One may operate only with the RAM (1K from $0-3776_{\theta}$ ) and PROM ( $1 \mathrm{~K}$ from $170000_{\theta}$ to $173776_{8}$ ) that reside on the LSI-11/2 processor board, or additionai memory may be plugged in. A fast (50-ns) iK board, and a medium speed (200-ns) 4 or $8 \mathrm{~K}$ board is provided to accommodate the dataacquisition users, while the slower 16K DEC dynamic memory board and a 4 or $8 \mathrm{~K}$ PROM memory board are available for control applications. A tape cassette unit and control panel have been designed for control applications, and will be described in a future paper dealing with stand-alone beam line control using the ACC. 


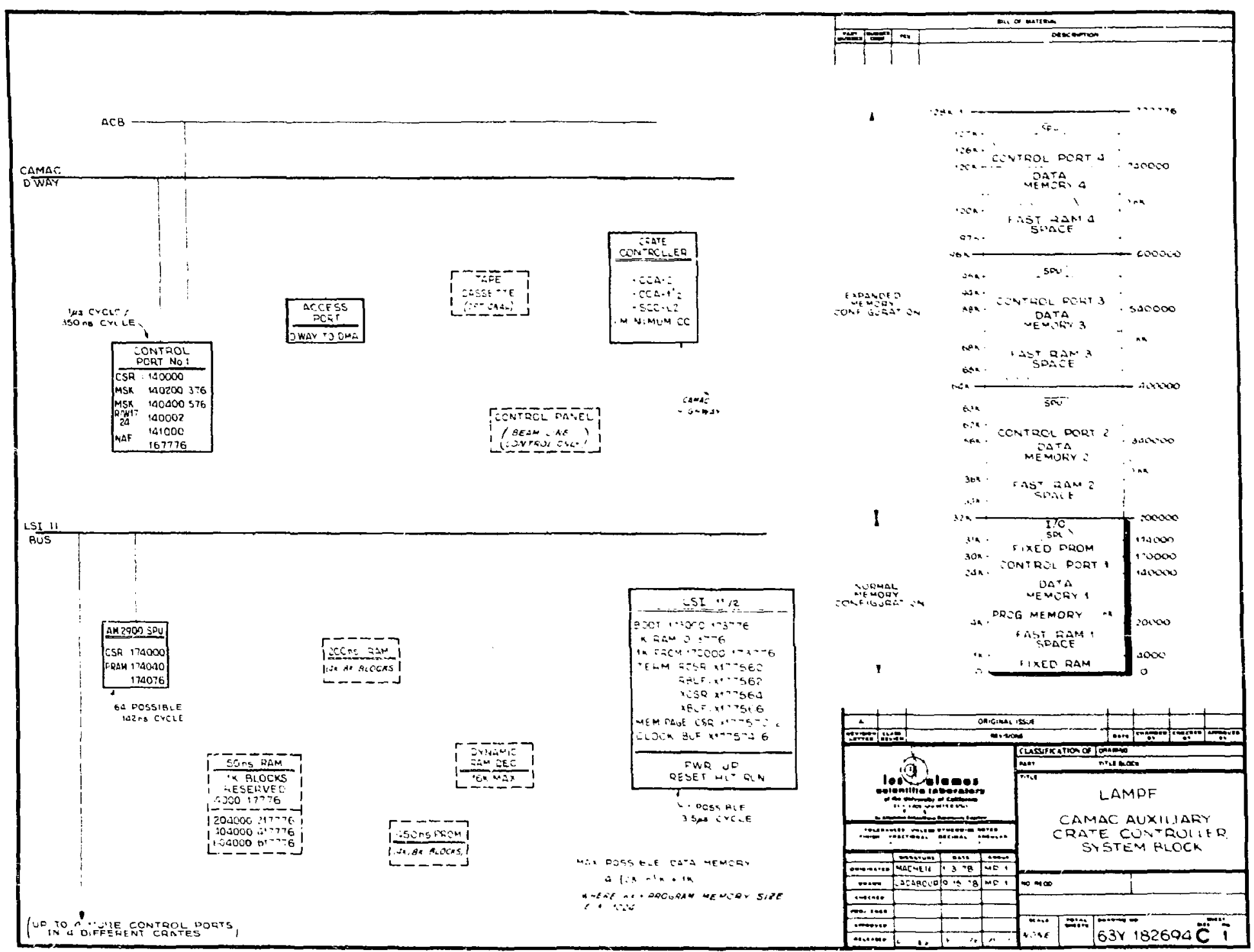

Fig. 2. Block diagram of ACC and address space allocation $(63 Y-182694 / 1)$. 
Figure 2 also shows the address space allocation for the LAMPF ACC. Logic has been included in the processor module to easily switch the address space to one of four $32 \mathrm{~K}$ segments. The purpose of this is not only to allow for a great deal of memory, but to easily accommodate multiple Control Ports and Special Processor Units (SPUS). The address space allocation indicates how fast ram and data memory could be spotted throughout the available 128K address space. Further, multiple Control Ports and multiple SPUs can be assigned address space in each $32 \mathrm{~K}$ address page.

Figure 3 indicates how, physically, the ACC extends to more than one CAMAC crate when an expanded dataway is required for some specific system probler:.

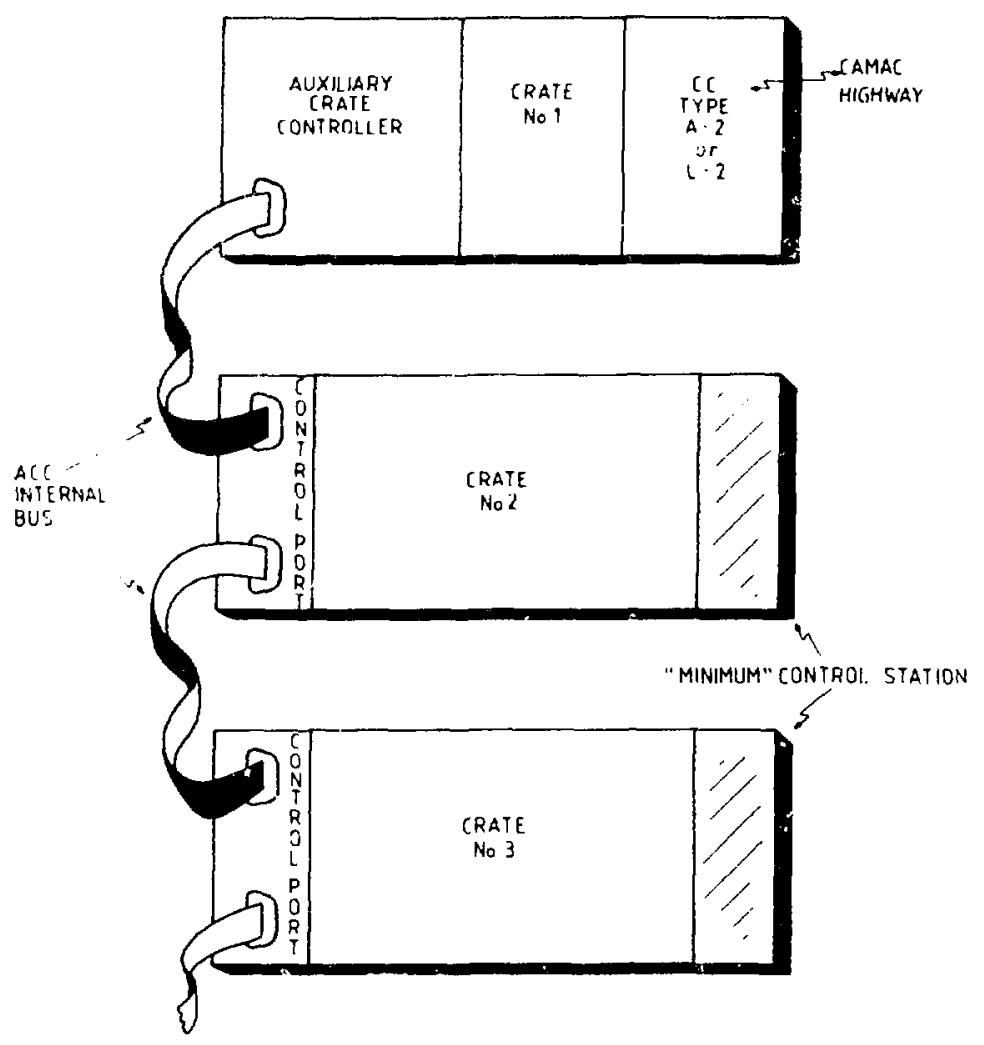

Fig. 3. Multicrate extension.

Note that the single-width Control Port interacts with a "minimal" controller only. The minimal controller consists of an N-Decode and L-patch in the simplest case, and an $\mathrm{N}$-Decoder plus L-grader in the more elaborate case. A description of the minimal controller is included at the end of Section II of this report.

Finally, a comment should be made concerning the selection of the LSI-11/2 as the primary processing elenients for the ACC. One could argue that nearly any single-chip microprocessor could be inccrporated successfully into the ACC; however we have found that a 16-bit word length machine has a significant advantage in speed and ease of programming over the various 8-bit devices on the market today. Further, LASL in general and LAMPF specifically uses a great number of 
the PDP-11 family computers. The software correspondence cannot be overlooked and was a major factor in selecting the $L \backslash I-11 / 2$ for use in the ACC.

\section{PROTOTYPE ACC BOARDS}

Each device noted in Fig. 2 making up the ACC will be described from its physical design standpoint in addition to its operational aspects. In the case of the AMD-2900 Special Processor Unit, the inicroinstruction set will also be covered.

\section{A. LSI-11/2 Processor Board}

This two-wide module is the heart of the ACC and contains sufficient hardware to operate in a stand-alone mode should a computing facility of limited capability be desired.

As viewed from the front panel, the LSI-11/2 resides on the left side, while the remainder of the board logic is on the right boarci as is the dual 43-pin card edge connector providing access to the Q-Bus. This connector is identical to the Dataway connector located in the rear of the card, but raised a few centimeters vertically from the Dataway connector. The power requirements are:

$\begin{array}{lll} & \underline{+5 \mathrm{~V}} & \underline{+12 \mathrm{~V}} \\ \text { Left side (LSI-11/2) } & 1.2 \mathrm{~A} & 0.22 \mathrm{~A} \\ \text { Right side } & 1.1 \mathrm{~A} & \\ \text { (This board also uses }-12 \mathrm{~V} \text { and }+24 \mathrm{~V} .)\end{array}$

The board contains the following manual controls:

RESET pushbutton - Causes the LSI-11/2 tc execute a Q-Bus INIT, and force a program jump to $173000_{8}$.

HALT/RUN - When this switch is in RUN, the $11 / 2$ is enabied to RUN on ly.

When the switch is in the HALT position, control will be transferred to microcode ODT. A terminal device must be connected to interact with ODT. See DEC manual for operating details.

BOOT MODE

- This switch controls the state of a status register bit to allow software coritrol of the boot-up program source. See terminal details.

Q-BUS CONNECTIONS TO ACC ERONT CONNECTOR

DEC Pin No.

AU2

AV2

BE2

BF2

$\mathrm{BH} 2$

BJ2

BK2
Signal

BDAL OL

$\begin{array}{ll}" & 1 L \\ " & 2 L \\ " & 3 L \\ " & 4 L \\ " & 5 L \\ & 6 L\end{array}$

ACC Connector No.

1 
(continued)

DEC Pin No.

BL2

BM2

BN2

$B R 2$

BS2

BT2

BU2

BV2

DEC Memory

on $7 y$

A $œ$ ?

$-$

$-$

$-$

$-$

-

-

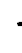

AJ2

AH2

AE2

AF2

AN1

$-$

AS2

A.L2

-

AN2

AP2

BNT

BR1

BA1

AP1

AT2

AHT
BP2
Signal

BDAL $7 L$

" $8 \mathrm{~L}$

" $9 \mathrm{~L}$

" $10 \mathrm{~L}$

" $11 \mathrm{~L}$

" $12 \mathrm{~L}$

" $13 \mathrm{~L}$

" $74 \mathrm{~L}$

" $15 \mathrm{~L}$

"16L $\}$ extended

" 17L address

BWTETL

SLI

SL2

$\mathrm{SL} 3$

SL4

SL5

SL 6

$+6 V, 2 A$

BSYNCL

BDINL

BDOUTL

BRPLYL

BDMRL

BDMGIL

BDMGOL

BIRQL

BIAKIL

BIAKOL

BBS7L

BSACKL

BEVNTL

BOCOKH

BHALTL

BINITL

RUNL
ACC Connector No.

\section{8}

9

10

11

12

13

14

15

16

?7 on ACC

18 boards

19

20

21

22

23

24

25

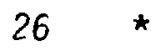

27

28

29

30

31

32

33

34

35

36

37

38

39

40

42

43

*Source from control port

**Boards not usin; DMA or INTERRUPT must jumper 32-33 and 35-36, respectively. 
DEC Pin No.

BMI AJI ALI

BTI AMT

AC2 AT1

$\mathrm{BC2}$ BJ1

BV1 BA2

AA2

$\mathrm{AD2}$

$\mathrm{BD} 2$
Signal

GROUND

$+5 V$

$+12 V$

\section{B. $\quad$ LS1-11/2 Board Hardware}

1. Fixed PROM and Boot Memory. A IK UV-er'asable, Programmable Read Only Memory (PROM) is avai Table at address:

$$
170000_{8} \text { to } 173776_{8}(30-31 K) \text {. }
$$

The bootstrap code chosen for a specific application must begin at $173000_{\theta}$ and may extend to the top of PROM. Again, the LSI-11/2 is set to transfer control to this boot location on RESET or power-up. The remaining free locations in on-board PROM may be used at the discretion of the system designer. Further, this PROM is only available on address page 0 ; the equivalent space on address page 1,2 , or 3 cåınot be assigned.

2. Fixed RAM. A $1 K$ Random Access Memory (RAM) is available at address:

0 to $3776_{8}(0-1 K)$.

NOTE: This memory may be disabled by switch option on the pc board.

As with the fixed PROM, this RAM is only available on address page 0 and the equivalent space on page 1, 2, or 3 cannot be assigned. Memory was included in the low end of address space in order that any given system will always have trap, interrupt, and stack space when needed. In addition, a certain amount of program space is available and may be used for small problems.

3. Memory Page Control. Control of the memory paging logic is available at Control/Status Register (CSR) address:

$$
\text { X.1775708, }
$$

where $X]$ indicates that the address responds for miemory page control on any page (i.e., $x_{1}=1,3,5,7$ ). 


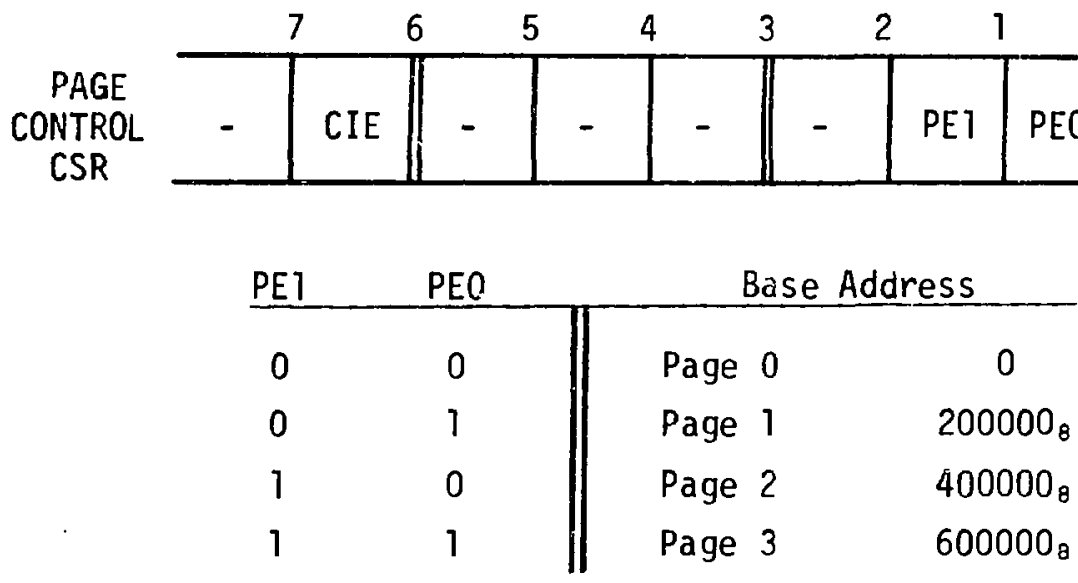

In order to conserve space on the processor module, the interrupt enable bit for the multifrequency clock was included in the CSR for the memory page logic. The reader is referred to the next section for the meaning of CIE. The base address is cleared to 0 on power-up or RESET.

4. Multifrequency Clock. A free-running, 16-bit multifrequency clock is available to be read at address:

$$
\times 177574_{8} \text {. }
$$

Again, $X$ indicates that the clock may be accessed at any page address. The clock frequency can be switch-selectable at $100 \mathrm{~Hz}$ or $1000 \mathrm{~Hz}$. The frequencies are generated by adjustable free-runnirg oscillators (VCOs) on the processor board. The clock is cleared to 0 on power-up or RESET.

Clock Interrupt--the $(100-\mathrm{Hz}$ or $1000-\mathrm{Hz})$ clock input can be routed to the LSI-11/2 EVENT Interrupt by setting CIE (bit 6) at $\times 177570_{8}$ to logic 1. Provided PS bit 7 is ciear (logic 0 ) and an appropriate vector is contained at address $100_{8}$ and $102_{\theta}$, the LSI-11/2 will interrupt to that vector on every clock tick. Alternatively, the clock may be read when desired to provide a measure of elapsed time.

5. Terminal Port. A multidevice terminal part is available on a rear 36-pin card edge connector. The connector is physically on the back of the rightmost $P C$ card when viewing the module from the front of the CAMAC crate.

The terminal port is configured to operate EIA standard terminals, a 20-mA teletype/paper tape, or devices using TTL levels. The port control is cleared on power-up or RESET.

- An 8-bit character may be read at address $X 177562_{8}$,

- An 8-bit character may be transmitted at address $x 177566_{a}$.

- A Receive CSR is avaijable at address $X 177560_{8}$. 


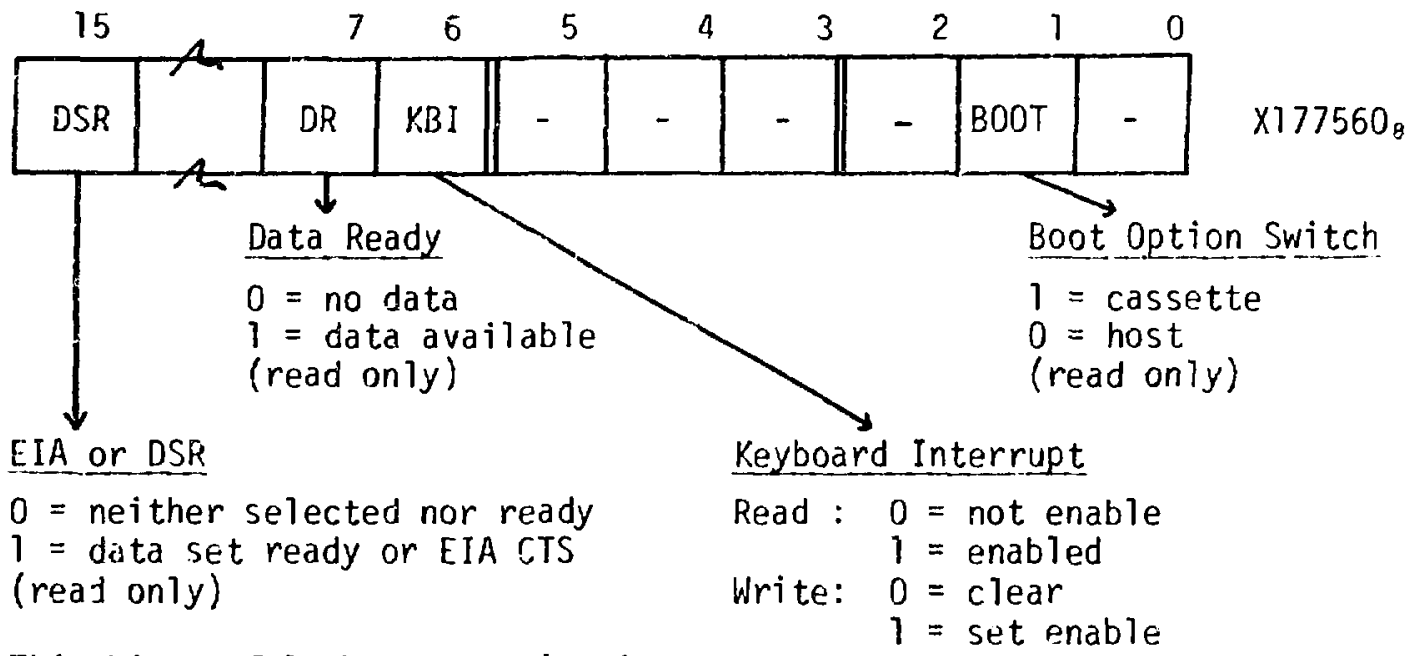

This bit useful when communicating through a modem.

NCTE: Unused bits are read as a logic 1.

A Transmit CSR is available at address $\times 177564_{\mathrm{B}}$.

Read only - bit 7 is Transmit buffer empty.

$$
\begin{aligned}
& 0=\text { furl } \\
& 1=\text { empty }
\end{aligned}
$$

NOTE: Unused bits are read as a logic 1.

Keyboard Interrunt to:

When enabled (bit 6 of RCSR $=1$ ), the LSI-11/2 nay receive an interrupt

Vector at $60_{\theta}, 62_{\theta}$,

provided the PS bit $7=0$ and the vector at $60_{8}$ to $62_{8}$ contains the proper information.

\section{Baud Rates}

The terminal port may be set to transmit and receive at a number of different baud rates set by switch located on the processor pc board.

\begin{tabular}{rrrrrr} 
Switch & 4 & 3 & 2 & 1 & Baud Rate \\
\hline 0 & 0 & 1 & 0 & 110 \\
0 & 1 & 0 & 1 & 300 \\
0 & 1 & 1 & 1 & 1200 \\
1 & 0 & 1 & 0 & 2400 \\
1 & 1 & 0 & 0 & 4800 \\
1 & 1 & 1 & 0 & 9600
\end{tabular}


The rear-mounted 36-pin edge connecter is wired in the following way:

\begin{tabular}{|c|c|c|c|c|c|}
\hline & & Fin & Signal & Pin & Signal \\
\hline IR & 1 & 1 & $20 \mathrm{~mA}$ out + & IR & $20 \mathrm{~mA}$ Out - \\
\hline $2 R$ & - . $\cdot 2$ & 2 & $20 \mathrm{~mA}$ In - & $2 \mathrm{R}$ & Ground \\
\hline $3 R$ & - 1. 3 & 3 & TTL Out & $3 R$ & " \\
\hline & & 4 & TTL In & $4 \mathrm{R}$ & $"$ \\
\hline & & 5 & EIA out & $5 R$ & " \\
\hline & & 6 & EIA In & $O R$ & $"$ \\
\hline & & 7 & EIA RTS/DTR & $7 R$ & $"$ \\
\hline Viewe & ved from & 8 & EIA CTS & $8 \mathrm{R}$ & $"$ \\
\hline rear & of module & 9 & EIA DSR & $9 \mathrm{R}$ & $"$ \\
\hline & & 10 & Reader Start + & 10R & Reader Start \\
\hline
\end{tabular}

The terminal port provides the following ASCII character handling:

1. Parity is neither generated nor checked.

2. Two stop bits are generated and checked.

3. Eight data bits are sent and received.

4. HALTL is asserted on a Frame Error.

C. Dataway Access Port

When an ACC is equipped wi th a Dataway Access Port, the system controller, via the Crate Controller and CAMAC Command Highway (see Fig. 1), is able to access memory and control the operation of the ACC. A Memory Address Register (MAR) and Memory Data Register (MDR) hold ACC bus cycle information during a Direct Memory Access (DMA) of the Q-bus. Owing to coupling latencies between the Dataway, the Q-bus, and the CAMAC Highway/System Controller, a requested Read or Write cycle is carried out when the Dataway cycle completes. For this reason, Read data must be acquired on the next CAMAC cycle addressed to the Access Port.

CAMAC Commands

The Access Port responds to a set of NAFs (1isted below) and will return $x=1$ to the system controller provided a command within the valid set is received.

Subaddress $A 0$.

DIS (F24) - Disable RUN and enter ODT.

XEQ (F25) - Bus INIT and Enable RUN.

The following notes pertain to the use of XEQ $A 0$ :

Condition Action Result

1. LSI-11/2

Issue Bus Init

not in ODT XEQ $\cdot A O$

Generated by Access Port

(no action to $11 / 2$, only others) 


$\begin{array}{lll}\text { Condition } & \text { Action } & \text { Result } \\ \text { 2. LSI-11/2 } & \text { Issue } & \text { Enable RUN only } \\ \text { is in ODT } & \text { XEO-AO } & \text { I1/2 Stays in ODT } \\ & & \text { (see XEO-AI to boot) }\end{array}$

Subaddress Al

$X E Q$

- Iritiates RESET to $11 / 2$ and forces program jur:s to $173000_{8}$ boot. $11 / 2$ issues BIIS INIT.

Subaddress A2

RO2 (FI) - Read MAR (RI6-RI)

WT2 (F17) - Write MAR (WI6-W1) and initiate DMA READ cycle at MAR.

$Q=1$ ACC DMA cycle able to uroceed.

$Q=0$ reisiue CAMAC, command as the DMA cycle was not able to proreed due to other ACC bus activity.

\section{Subaddress $A 3$}

RDI (FO) - Read MDR (RI6-RI)

This command must follow a successful TST.A4 to ensure that a previous read request took place.

WTI (F16) - Write MDR (W16-W1) and initiate a DMA WRITE cycle to the ACC bus.

$Q=1$ MDR write took place and DMA cycle able to proceed. Following the DMA cycle MAR \& MAR+2.

This command must be followed by a TST.A4 to determine when the DMA cycle is complete.

XEQ - MAR - MAR+2 then initiates a DMA READ cycle to the ACC bus.

$Q=1$ increment took place and the OMA cycle is able to proceed.

This command musr also be followed by a TST.A4 to determine whon the DMA cycle is complete.

Subaddress A4

TST (F27) $-Q=1$ indicates that the requested cycle took place and that no other device request is pending. 
Subaddress A12

$\mathrm{RO2}$

- Read the ACC status register (R3-R1)

RI $=1$ Dataway Access Port cycle in progress.

R2 - I Another ACC device is bus master.

R3 $=$ I HALT asserted (either by Access Port logic or by switch on LSI-11/2 module).

Dataway C. $\$ 2$ generates an internal Access Port reset and $Z$ is not responded to.

\section{Operating Philosophy}

Since Read and Write operations are accomplished by DMA bus sharing, it is quite possible for the System Controiler to read-out or load a section of ACC Memory while other operations are under way. I would caution the user, however, to halt ACC activity if a great deal of interrupt or other DMA activity is going on. This activity will only show the Access Port response and create a latency that may not be acceptahle. Provided the user knows what the ACC is up to, shared Access Port operation is permissible.

I alsc caution the user in the careful use of the various $Q$ tests and the appropriate application of the TST.A4 command. Always check Q and issue the TST command at the required intervals when executing Read and Write ACC bus cycles.

\section{ACC Memory}

The various memory options available have been discussed in part in Section I of this report. In this section, the address compare switch settings will be presented, along with some comments concernirg the types of memory and their ultimate use in ACC systems.

One additional memory should be mentioned as it. will find use in certain control applications of the ACC. This is the DEC MSV-17-DC 16K MOS Dynamic RAM. The memory is packaged on a 1-wide CAMAC moduie with a single DEC connector inside the module for the memory board, and a 43-pin ACC bus connector available at the front of the module. The unit draws $1.7 \mathrm{~A}$ at $+5 \mathrm{~V}$ and $0.34 \mathrm{~A}$ at $+12 \mathrm{~V}$. Five switches are located on a "DIP Switch" near the board connector, as shown below. 
A "closed" switch $=0$ or on, and an "open" switch $=1$ or off.

\begin{tabular}{cccccc} 
& \multicolumn{5}{c}{ Switch No. } \\
Starting Address & 1 & 2 & 3 & 4 & 5 \\
20040 & 0 & 0 & 0 & 0 & 1 \\
40000 & 0 & 0 & 0 & 1 & 0 \\
60000 & 0 & 0 & 0 & 1 & 1 \\
100000 & 0 & 0 & 1 & 0 & 0 \\
120000 & 0 & 0 & 1 & 0 & 1 \\
140000 & 0 & 0 & $?$ & 1 & 0 \\
160000 & 0 & 0 & 1 & 1 & 1 \\
200900 & 0 & 1 & 0 & 0 & 0 \\
220000 & 0 & 1 & 0 & 0 & 1 \\
240000 & 0 & 1 & 0 & 1 & 0 \\
260000 & 0 & 1 & 0 & 1 & 1 \\
300000 & 0 & 1 & 1 & 0 & 0 \\
320000 & 0 & 1 & 1 & 0 & 1 \\
340000 & 0 & 1 & 1 & 1 & 0 \\
360000 & 0 & 1 & 1 & 1 & 1 \\
400000 & 1 & 0 & 0 & 0 & 0 \\
420000 & 1 & 0 & 0 & 0 & 1 \\
440000 & 1 & 0 & 0 & 1 & 0 \\
460000 & 1 & 0 & 0 & 1 & 1 \\
500000 & 1 & 0 & 1 & 0 & 0 \\
520000 & 1 & 0 & 1 & 0 & 1 \\
540000 & 1 & 0 & 1 & 1 & 0 \\
560000 & 1 & 0 & 1 & 1 & 1 \\
600000 & 1 & 1 & 0 & 0 & 0 \\
620000 & 1 & 1 & 0 & 0 & 1 \\
640000 & 1 & 1 & 0 & 1 & 0 \\
660000 & 1 & 1 & 0 & 1 & 1 \\
700000 & 1 & 1 & 1 & 0 & 0
\end{tabular}

This memory must not be started at 0 , as RAM already exists from 0 to $3776_{8}$ on the LSI-11/2 board, unless that RAM has been disabled by switch option.

200-ns RAM

In applications where a faster memory cycle time is required and/or the random latencies generated by the dynamic RAM refresh operation cannot be tolerated, a 4 to $8 \mathrm{~K}$ (depending upon the number of memory chips plugged in) static RAM is available. The meliory contains AMD-9130 1024 x 4 static RAM chips ( 16 for $4 \mathrm{~K}$ or 32 for $8 \mathrm{~K}$ ) and can be loaded with any multiple of $1 \mathrm{~K}$ words as long as the system application can accept a $4 \mathrm{~K}$ or $8 \mathrm{~K}$ block address space assignment independently of the actual amount of RAM implemented.

An 8-switch "DIP-switch" is located on the module from which six switches are used to select the starting adoress and the $4 \mathrm{~K}$ or $8 \mathrm{~K}$ option. Again, a closed switch, or on, = logic 0 and an open switch, or off, = logic 1 . Switch 6 controls the $4 \mathrm{~K}$ to $8 \mathrm{~K}$ option. The unit is a 1 -wide module, drawing $1.8 \mathrm{~A}$ at $+5 V$ for the $4 K$ option. 


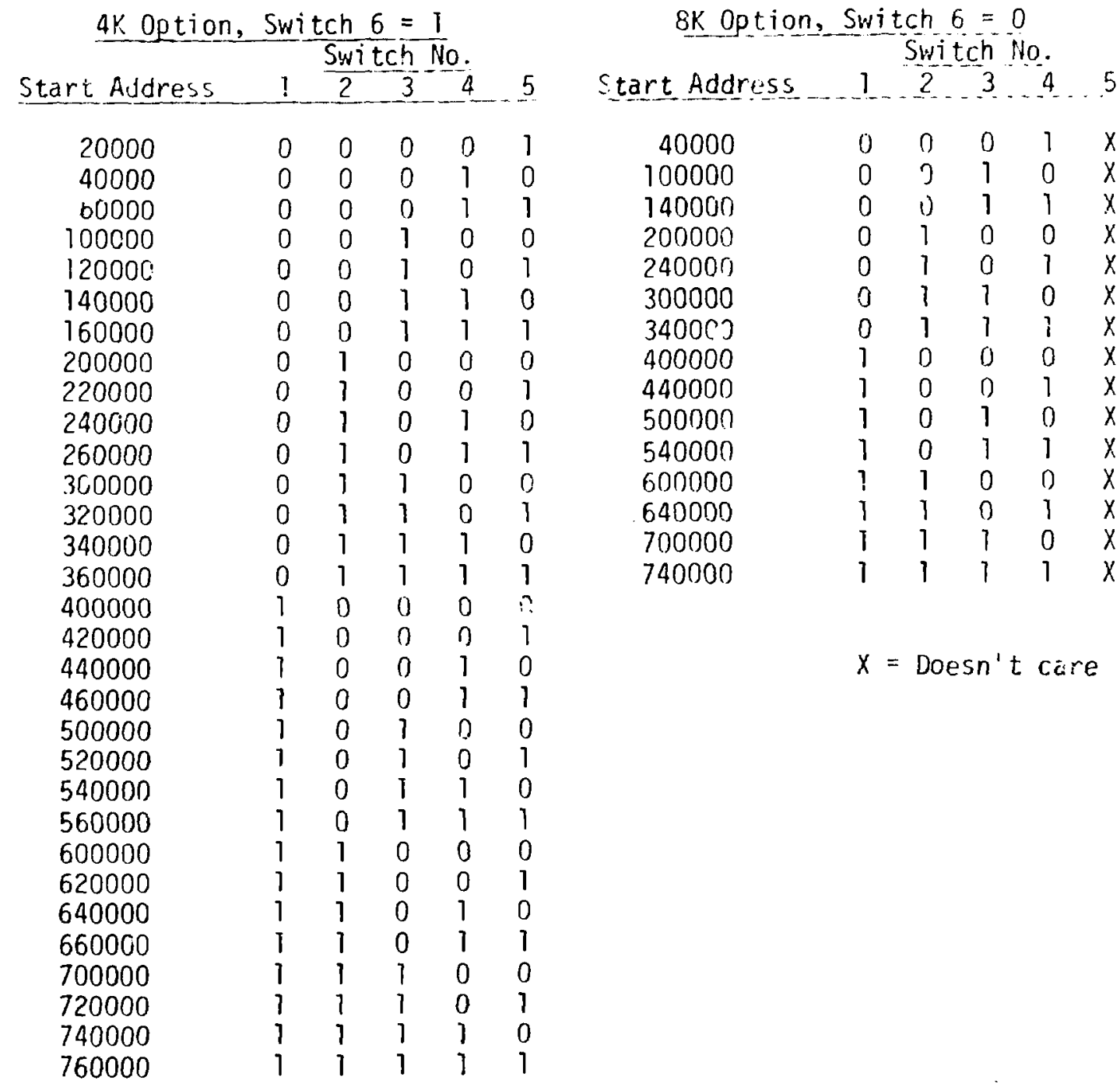

50-nS PAM

A $1 K$. 50-ns fast RAM has been designed using a $1 K \times 1$ Fairchild $93 L 425$ static RAM chip. This memory will find use in ACC front-end data acquisition and filtering applications where fast buffer store is required.

The unit is a 7 -wide CAMAC module requiring $1.2 \mathrm{~A}$ at $+5 \mathrm{~V}$.

An 8-switch "DIP-switch" is located or the module from which five switches are used to select the starting address, which cail be spotted in either low or high memory in any of the four address pages. A closed switch, or on, = logic 0 , anc an open switch, or off, $=$ logic 1 . Switches 1 and 2 control the page boundary, switch 3 controls low or high memory placement, ana switches 4 and 5 control the $1 \mathrm{~K}$ boundary. Again, this memory should not be placed at address 0 , as memory at that address a Tready exists on the LSI-11/2 board, unless the address 0 RAM has been disabled by switch option. 


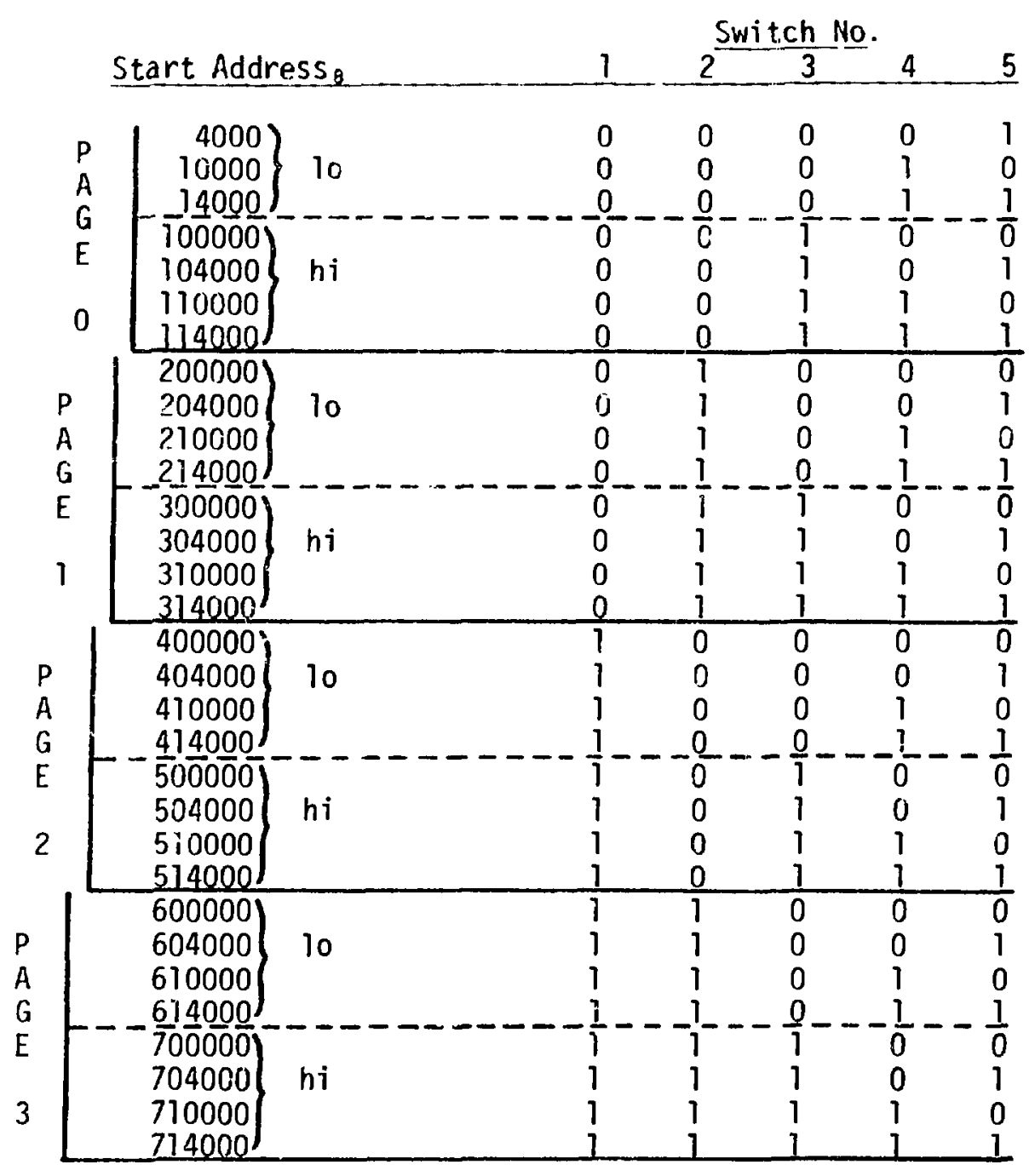

The ieader is referred to Fig. ", address space allocation, to see graphically where the $I K$ fast RAM may be placed.

450-ns PROM

Applications involving the ACC in stand-alone or partially stand-alone control systems will dictate that checked-out code be placed in nonvoiatile read only memory. In order that a small quantity of this menory be economically feasible and practical for laboratory use, an ultra violet erasable PROM (Intel $2716,2 \mathrm{~K} \times 8$ ) was selected. The cycle time is $450 \mathrm{~ns}$ and the $4 \mathrm{~K} / 8 \mathrm{~K}$ memory is constructed on a 1 -wide CAMAC module, drawing $1 \mathrm{~A}$ at $+5 \mathrm{~V}$. Programing of the PROM chip is accomplished on any of the microprocessor development machines available at LAMPF.

An 8-switch "DIP-Switch" is located on the module from which six switches are used to select the starting address and the $4 \mathrm{~K}$ or $8 \mathrm{~K}$ option. The closed, or on, switch = logic 0 , and the open, or off, switch = logic 1. Switch 6 controls the $4 \mathrm{~K} / 8 \mathrm{~K}$ option. 
4K Option, Switch $6=1$

\begin{tabular}{|c|c|c|c|c|}
\hline \multirow{2}{*}{ Start Address } & \multicolumn{4}{|c|}{ Switrh N } \\
\hline & 1 & 2 & 3 & 4 \\
\hline 20000 & 0 & 0 & 0 & 0 \\
\hline 40000 & 0 & 0 & 0 & 1 \\
\hline 60000 & 0 & 0 & 0 & 1 \\
\hline 100000 & 0 & 0 & 1 & 0 \\
\hline 120000 & 0 & 0 & 1 & 0 \\
\hline 140000 & 0 & 0 & 1 & 1 \\
\hline 160000 & 0 & 0 & 1 & 1 \\
\hline 200000 & 0 & 1 & 0 & 0 \\
\hline 220000 & 0 & 1 & 0 & 0 \\
\hline 240000 & 0 & 1 & 0 & 1 \\
\hline 260000 & 0 & 1 & 0 & 1 \\
\hline 300000 & 0 & $?$ & 1 & 0 \\
\hline 320000 & 0 & 1 & 1 & 0 \\
\hline 340000 & 0 & 1 & 1 & 1 \\
\hline 360000 & 0 & 1 & 1 & 1 \\
\hline 400000 & 1 & 0 & 0 & 0 \\
\hline 420900 & 1 & 0 & 0 & 0 \\
\hline 440000 & 1 & 0 & 0 & 1 \\
\hline 460000 & 1 & 0 & 0 & 1 \\
\hline 500000 & 1 & 0 & 1 & 0 \\
\hline 520000 & 1 & 0 & 1 & 0 \\
\hline 540000 & 1 & 0 & 1 & 1 \\
\hline 560000 & 1 & 0 & 1 & 1 \\
\hline 600000 & 1 & 1 & 0 & 0 \\
\hline 620000 & 1 & 1 & 0 & 0 \\
\hline 640000 & 1 & 1 & 0 & 1 \\
\hline 660000 & 1 & 1 & 0 & 1 \\
\hline 700000 & 1 & 1 & 1 & 0 \\
\hline 720000 & 1 & 1 & 1 & 0 \\
\hline 740000 & 1 & 1 & 1 & \\
\hline 760000 & 1 & 1 & 1 & 1 \\
\hline
\end{tabular}

8K Option, Switch $6=0$

Switch No.

Start Address $1 \frac{1}{2} 3 \quad 4 \quad 5$

$\begin{array}{llllll}40000 & 0 & 0 & 0 & 1 & x \\ 100000 & 0 & 0 & 1 & 0 & x \\ 140000 & 0 & 0 & 1 & 1 & x \\ 200000 & 0 & 1 & 0 & 0 & x \\ 240000 & 0 & 1 & 0 & 1 & x \\ 300000 & 0 & 1 & 1 & 0 & x \\ 340600 & 0 & 1 & 1 & 1 & x \\ 400000 & 1 & 0 & 0 & 0 & x \\ 440000 & 1 & 0 & 0 & 1 & x \\ 500000 & 1 & 0 & 1 & 0 & x \\ 540000 & 1 & 0 & 1 & 1 & x \\ 600000 & 1 & 1 & 0 & 0 & x \\ 640000 & 1 & 1 & 0 & 1 & x \\ 700000 & 1 & 1 & 1 & 0 & x \\ 740000 & 1 & 1 & 1 & 1 & x \\ & & & & & \\ & & & & & \end{array}$

E. Control Port

The ACC Control Port (CP) performs CAMAC Dataway command operations in response to addressed DATI (Read) and DATO (Write) Q-bus cycles. A Status Register controls the mode of operation with respect to Dataway cycles and LAM response. A normal LAM source has been provided which can be enabled from the Dataway (by the System Controller) and set by the ACC. This feature allows an ACC to gain the attention of the system controller in an assynchronous way for the purpose of passing inforination or warning messages, etc.

The user must be familiar with the connection options for priority arbitration of Dataway Mastership. The LAMPF ACC follows the requirements of DOE/EV-0007 for generation and reception of Request/Grant signals and reception of the Auxiliary Controller Lockout signal.

A 4-switch "DIP-Switch" is located on the module from which two switches are used to select the page boundary address of the CP. The closed, or on, switch = logic 0 , and the open, or off, switch = logic l. 


\begin{tabular}{lcc} 
& \multicolumn{3}{c}{ Switch No. } \\
CP Address Range & 1 & 2 \\
\hline $140000-167776$ & 0 & 0 \\
$340000-367776$ & 0 & 1 \\
$540000-567776$ & 1 & 0 \\
$740000-767776$ & 1 & 1
\end{tabular}

using the boundary setting switches, one can place up to four coritrol ports with one,$C C$, one CP per CAMAC crate, and effectively exterid the primary crate space. at +5 V.

The Control Port is constructed on a 7 -wide CAMAC module and reguires $1.48 \mathrm{~A}$

CAMAC NAF

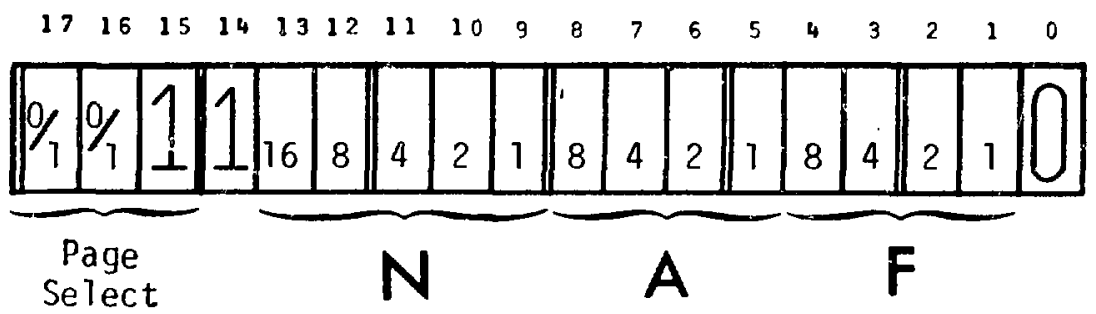

The address allocation for a Mapped CAMAC NAF, with Nø and N24-N3i being illegal addresses within a CAMAC crate, is:

$$
X 141000_{3}-X 167776_{8} \text {. }
$$

The mapped address to NAF table then looks like:

$$
\begin{array}{cc}
\text { N1AOFO } & \rightarrow \text { X141000 } \\
\text { N1AOF } i & \rightarrow \text { X141002 } \\
\vdots & \vdots \\
\text { N23A15F15 } & \rightarrow \text { X167776 } .
\end{array}
$$

\begin{tabular}{|c|c|c|}
\hline Q-Bus Cycle & $F(8)$ & Datawoy Operation \\
\hline $\operatorname{CATI}_{\|}(\mathrm{READ})$ & $\begin{array}{l}0 \\
1\end{array}$ & $\begin{array}{ll}\text { F0-F7 } & \text { READ } \\
\text { F8-F15 } & \text { CONTROL }\end{array}$ \\
\hline DATO (WRITE) & $\begin{array}{l}0 \\
1\end{array}$ & $\begin{array}{l}\text { F16-F23 WRITE } \\
\text { F24-F31 CONTROL }\end{array}$ \\
\hline
\end{tabular}

The following Q-bus cycles, along with $F(8)$ bit, providc for the switching between the various types of CAMAC functions:

DATI bus cycles to the CP (F0-F7) access the CAMAC R lines R16-R1, and latch R24-R17 into a holding register for later access. DATI bus cycles to the CP (F8-F15) transfer $Q$ on bit 0 and $X$ on bit 1 for the Dataway cycle executed. 
DAT0 bus cycles to the CP (F16:F23) will transfer bus data to W16-W1 and the contents of the W24-W17 holding latch to W24-W17. A DAT0 bus cycle to the CP (F24-F31) will only execute the Dataway cycle, and Q/X for that operation must then be read from the $\mathrm{CF}^{\prime}$ Status Register.

R/W 17-24 Holding Register

The high-order 8 bits associated with the Read and Write Dataway Operation are accessed as follows:

\section{Q-BUS CYCI.E}

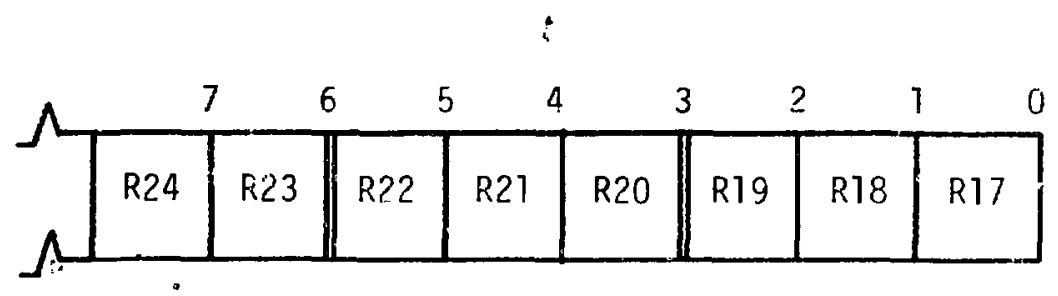

ALDRESS

DATI

(READ)

The R24-R17 lines are held during a READ CAMAC operation and may be read-out after the cycle is complete.

DATO

(WRITE)

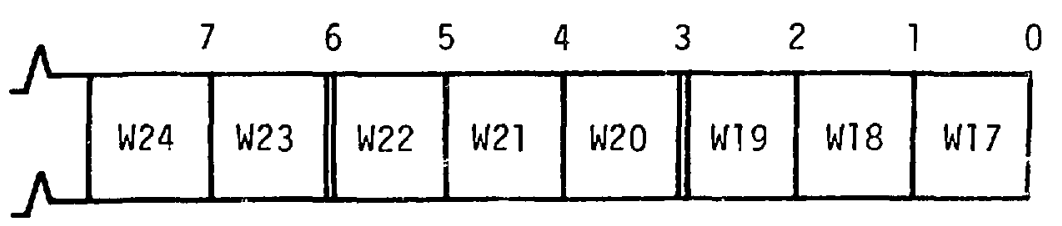

The W24-W17 buffer must be written prior to the WRITE CAMAC Operation, if these lines are used in the transactinn

Example: MOV O\#141000,RO; N1AOFO 10 byte

and

MOV RT,Q\#140002 ; WRITE hi byte

MOV PIO, @\#141000; NIAOF16

The CP Status Register

The Status Register can be accessed at address:

$\times 1: 40000_{8} \quad$,

and is useful in controlling the operational mode of the $C P$, in acidition to providing access to some special features of the LAMPF ACC.

DATI

(READ)

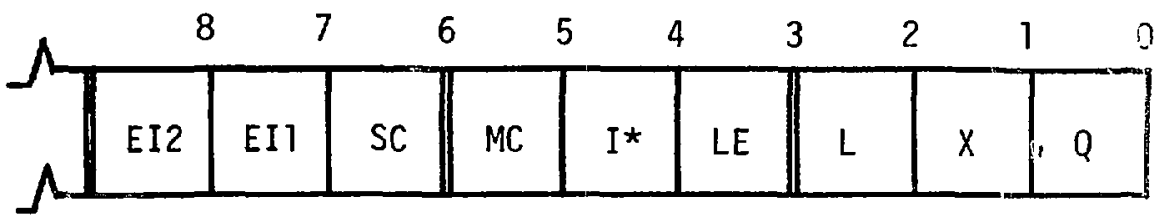

$\mathrm{X} 140000_{8}$ 
Bits $9-j 5$ are read as 15 .

$Q$ and $X$ are from the last Dataway cycle executed.

$L$ is the state of the CP L-Source.

LE is the state of the CP L-Mask.

I* is the state of Dataway Inhibit.

$M C$ is the state of the Multi-cycle mode control.

SC is the state of the Short-cycle mode control.

EI1 is the state of the $11 / 2$ interrupt logic enable.

EI2 is the state of the SPU LAM bus enable.

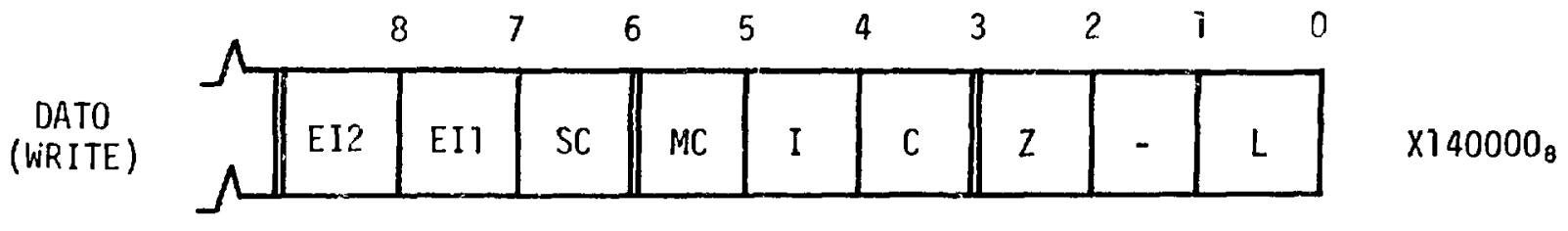

Bits $9-15$ are not used.

$L=1$ sets the CP L-Source to logic 1 .

$Z=1$ initiates a Dataway $Z$.

$C=1$ initiates a Dataway $C$.

NOTE: Both $Z$ and $C$ must be executed in normal cycle mode. The CP lagic momentarily sets normal cycle during the period of these commands, and returns to short-cycle mode if that mode had been invoked prior to $Z$ or $C$ being given.

$I=1$ sets and holds the Dataway Inhibit line.

$M C=1$ sets Multi-cycle mode.

$\mathrm{SC}=1$ sets Short-cycle mode.

$E I 1=1$ enables the $11 / 2$ interrupt logic.

$\mathrm{EI} 2$ = 1 enables the SPU LAM bus.

Multi-Cycle Mode

An Auxiliary Controller is allowed to execute multi-cycle control of the Dataway provided all other priority arbitration ru?es are met (see DOE/EV-0007). In multi-cycle mode, the CP initially arbitrates for Dataway Mastership preceding the first Dataway cycle. Once Dataway Busy is asserted, it remains asserted until Multi-cycle mode is cleared and the control port then executes single-cycle operations. An ACC operating in Multi-cycle mode has fewer overhead latencies per Dataway cycle and is, therefore, somewhat faster than one operating in single-cycle mode.

\section{Short-Cycle Mode}

The LAMPF ACC is capable of executing 350-ns Dataway cycies once SC =: 1. This cycle does not contain strobe S2, and all other times (see Fig. 9 of IEEE Standard 583-1975) are considerably shortened. The following sketch indicates the Dataway timing for the SC mode.

Clearly, a CAMAC module accessed by the CP in Short-cycle mode must be able to respond in the times indicated above. This mode is useful in high data-rate applications. 


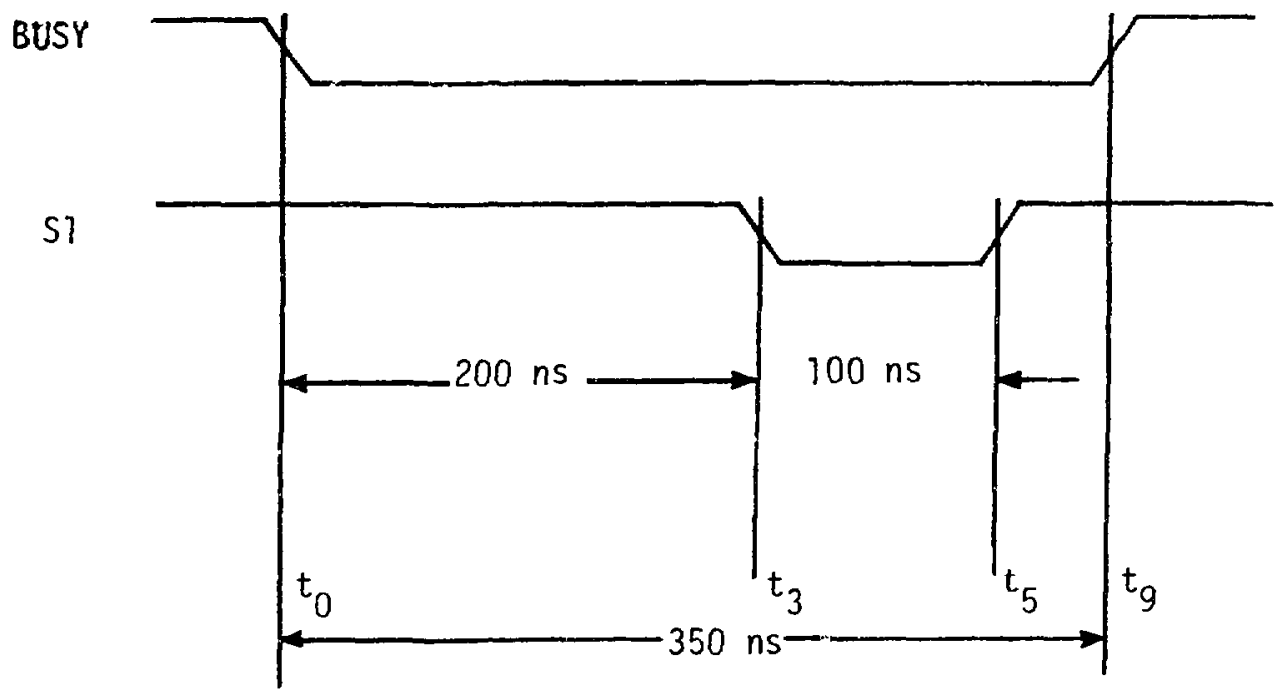

LAM Handling

The CP provides two parallel paths for coping with LAMS within the crate:

- One path, enabled by EII in the Status Register, priority encodes 23 LAM lines (L1 - highest priori $\forall \ddot{y}, L_{23}=$ lowest 3 riority) and presents the resultant 5 -bit code to the LSI-11/2 as an interrupt vector address. Base Address $=400_{8}$.

- The second path, enabled by EI2 in the Status Register, places up to six selected LAMS on a special set of six lines in the expanded ACC Q-bus.

These lines can be tested by a Special Processor Unit (SPI!) for use in highspeed data system requirements. More will be said about this technique in Section IIF.

A RAM mask provides the CP masking for LSI-11/2 interrupts. A $64 \times 1$ RAM is available at address:

$$
X 140200_{8}-X 740376_{8}
$$

Cleaily, only the first 24 locations are of concern, as the RAM is addressed by the encoded LAM pattern of the crate. However, the other 40 locations can be used as a 1-bit store for special code, as required by the user.

Prior to writing the mask, EI1 must $=1$.

- A DATO cycle to X1402\%X will write the state of bit 1 into the RAM at location $X X$. A logic 1 enables the corresponding LAM.

- A DATI cycle to $X 1402 \times X$ will read the state of location $X X$ into bit 1 of the destination.

The following table contains the correspondence between LAM-Mask Address and $11 / 2$ vector for the first LAM handling scheme. 


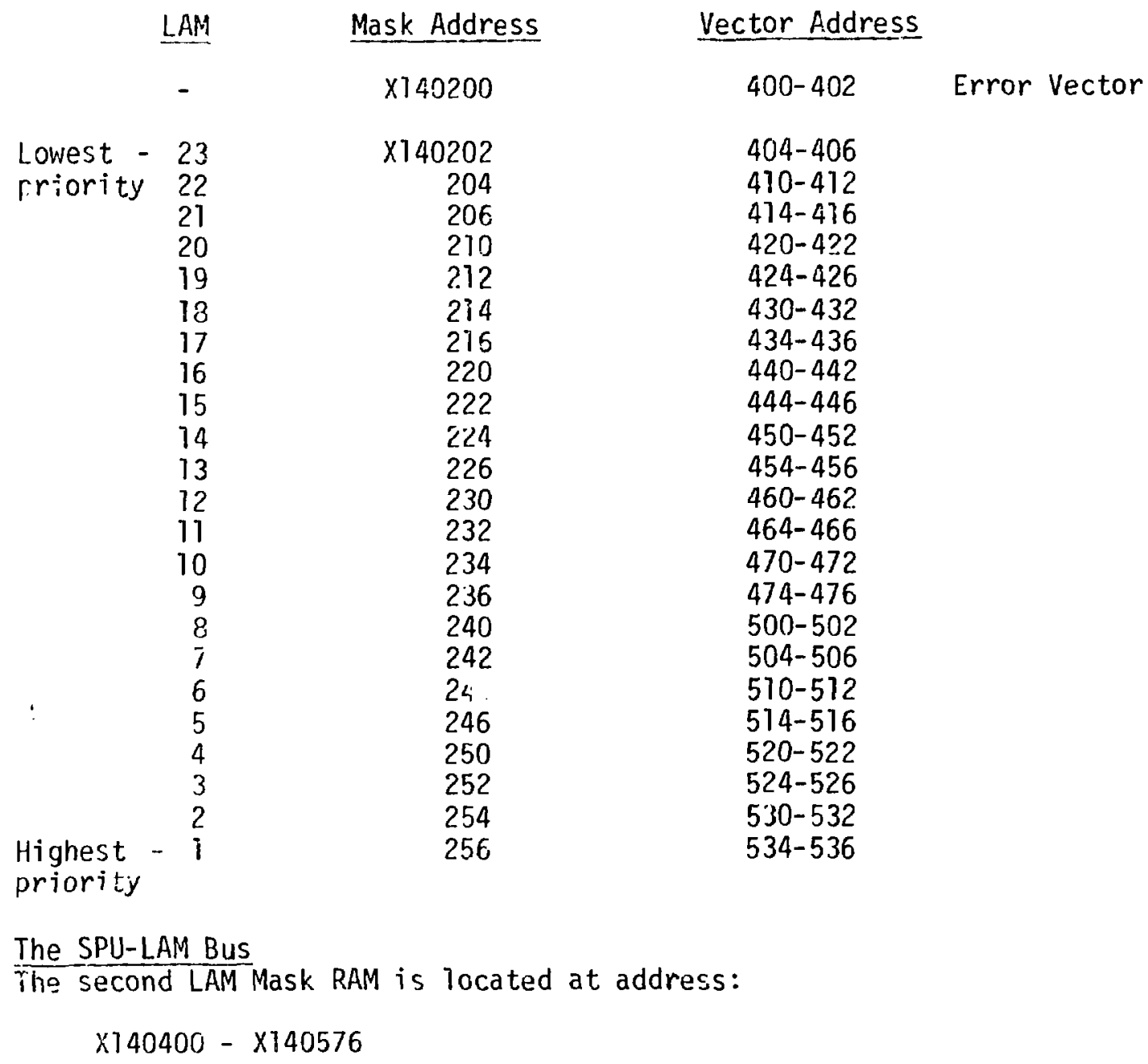

and is a $64 \times 1$ memory that is addressed by six patchable LAMS selected by the user. Further, the user must decide what combinations of the six selected (or less) LAMS will be masked on. If a given combination of LAMS is enabled (that address contains a logic 1), then the six LAMS are placed on six special Q-Bus LAM lines. The lines will be asserted within 100 ns of the assertion of ary particular L.AM.

Prior to writing the Mask, EI2 must $=1$.

- A DAT0 cycle to X7404a? will write the state of bit 1 into the RAM 2 at location XX. A logic 1 enables the corresponding LAM pattern.

- A DATI cycle to $X 1404 \times X$ will read the state of location $X X$ to bit 1 of the destination.

Switch 4 of the CP Address select "DIP-Switch" is an enable/disable control for the RAM2.

An Open SW4 = Normal operation as above.

A Closed SW4 = All 6-bit patterns enabled. 
The CP LAM Source

A System Controller, via a Crate Controller in Station 23-24 (or the CP itself), may set up a LAM from the ACC for use in Demand communications with a System Controller. The LAM Mask is addressed via the Dataway at Subaddress 0 and the station number associated with the $\mathrm{CP}$.

\begin{tabular}{|c|c|c|}
\hline address AO: & $\begin{array}{l}\text { ENB (F26) } \\
\text { DIS (F24) } \\
\text { CLM (F10) } \\
\text { TLM (F8) }\end{array}$ & $\begin{array}{l}\text { Enable the LAM } \\
\text { Disable the LAM } \\
\text { Clear the Source } \\
\text { Test the LAM independent of the Mask state }\end{array}$ \\
\hline
\end{tabular}

$X$ is returned for each command above.

The LAM-source is controlled by bit - 0 of the CP Status Register in a DATO cycle. Bit 3 of the CP Status Register during a DATI cycle contains the state of the LAM Mask. Thus, once set up, the ACC can send a Demand to a System Controller.

F. The Special Processor Unit useful hardware to further a given (larger) task, is to utilize the newly available state-of-che-art technology and not to reinvent a wheel. To this end, the 16-bit LSI-11 microcomputer was chosen as the primary control e? software compatibility and a reasonable word size were prime factors in the choice (the Ferrenti F100L was chosen for the Daresbury unit for those same reasons at that laboratory).

From the point of view of program execution times, the [SI-11 is no better than other minicomputers. The question which remained was how to improve the overall execution times of a certain subset of available ACC operations. One answer to this question was to utilize the newly available bit-sliced, bipolar microprocessor technology in a microprogramned subroutine structure which would be capable of operating at better than 200 ns per instruction cycle time. The AML-2900 family of LSI chips was selected for this purpose, and a microprogramming structure which ailows both register trarisfer operations and a reasonatle level of arithmetic capability was designed. The organization and operation of the Special Processor Unit (SPU), as it is called, will be described in this section.

The SPU Organization

The block diagram of the SPU is contained in Fig. 4 and shows how a microcoded controller, Arithmetic Logic Unit (ALU) and bus interface combine to form a fast processing facility capable of using the Q-Bus and, therefore, all devices in an ACC.

The SPU is a 1-wide CAMAC module with Q-Bus front connector and requires $2.95 \mathrm{~A}$ at $+5 \mathrm{~V}$.

SPU Operation

It is strongly recommended that the reader review the AMD-2900 family technical literature before attempting to go much further in this section. Although the finite state machine (constructed from LSI circuits available from AMD) is not especially complex from an outside view, the overall complexity is enormous! The machine is sssentially the control unit of a full-scale computing facility, with a 16-bit ALU added to allow logical and arithmetic operations to be 


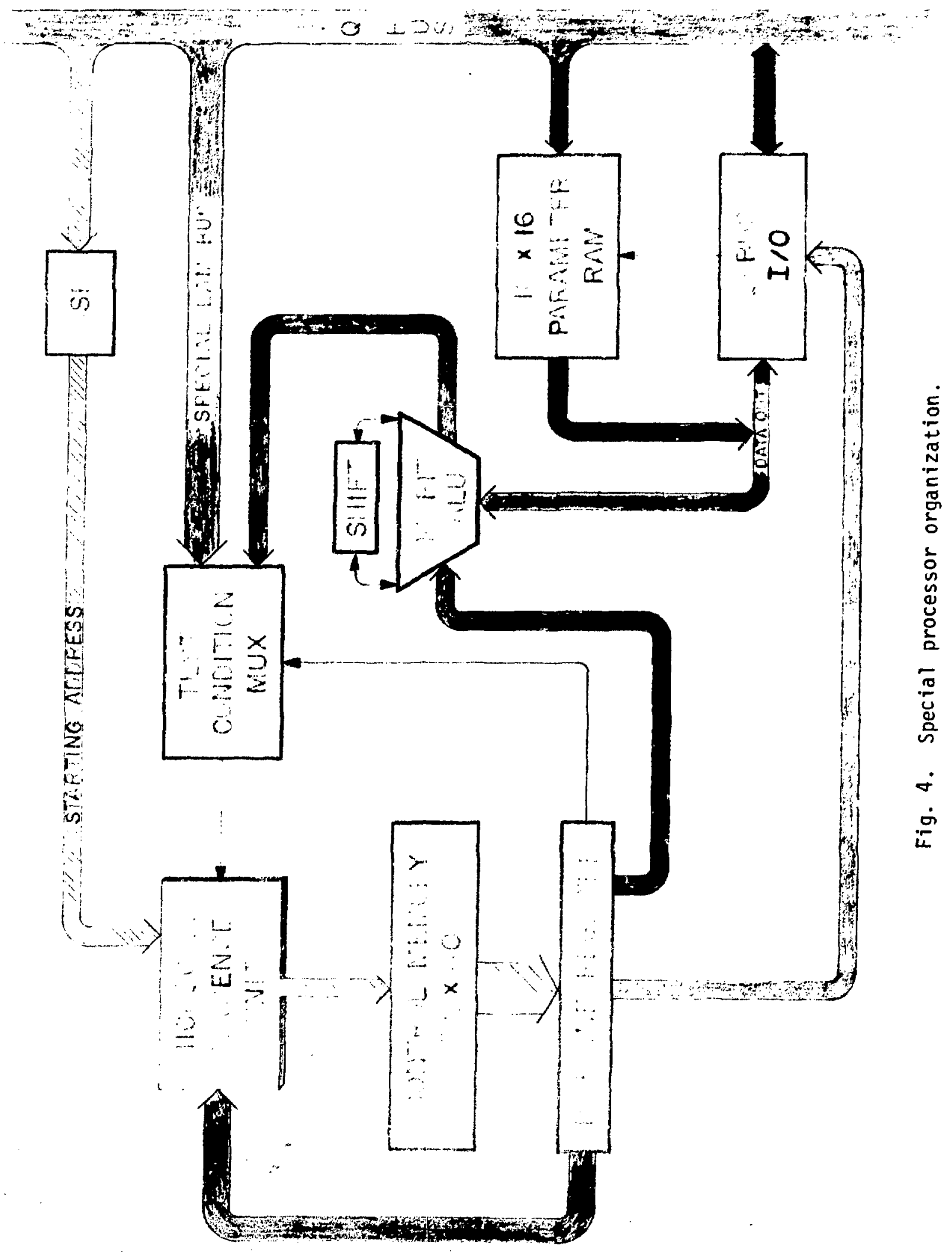


accomplished. The LSI technology which makes such a structure feasible is the 2910 micro-sequencer/address control unit. The 4-bit-wide ALUs (2901A) are designed to operate with the 2910 , but are not really special from the point of view of a micro-coded controller. The four ALU slices could just as well have been used with a hardwired control sequencer, and not the firmware controller designed around the 2910.

The finite state machine cycles with a 142-ns period (7-MHz clock) and is synchronous with the rising edge of the clock. The clock Juty factor is, by design, 40 to $42 \%$ in order to allow more signal set-up time at various chip inputs.

The control memory consists of five $512 \times 8$ fuse-link PROMs to make up the $512 \times 40$ memory. Fuse-link PROMs were selected for the ir fast access time (45 ns), and are considered essential in such a design. The control sequencer is capabie of generating a 12-bit "next-address" of which we make use of 9 bits and could expand to 11 bits.

Expansion of the control memory and/or testing of the SPU by PROM simulation has been provided for by two 3M-type connectors on the SPU PC board. A 20-pin connector brings the PROM address plus extended address bits out, and a 40-pin connector brings the 40 micro-instruction bits in. See section IIs for a description of the simulator designed to work into these connectors, in addition to a memory expansion option.

The contents of the controi memory (the micro-instructions) are applied to a 40-bit "pipeline" register (PLR) which is clocked on the rising edge of the 7-MHz clock. All machine control is derived from various signals at the output of the PLR. The theory behind a PLR in a finite state machine design is to allow the sequence unit and control memory to settle to the next address during the execution at the current address, thus speeding the instruction time by that settling and access time.

One field of the micro-instruction is a function control to the sequence unit. The instructions will be discussed in Section IIIB, but it is sufficient to say, at this point, that the generation of the next instruction address is controlled through this function code. The next address may be conditional upon some number of test conditions; a field in the micro-instruction word selects these conditions. Finally, the state machine must be told what state to begin execution in; the 11-bit direct input from a Control/Status Register (accessed via the Q-Bus) provides this information. 
The CSR is available at address:

$$
\times 174000_{a} \text {, }
$$

and the functions available are:

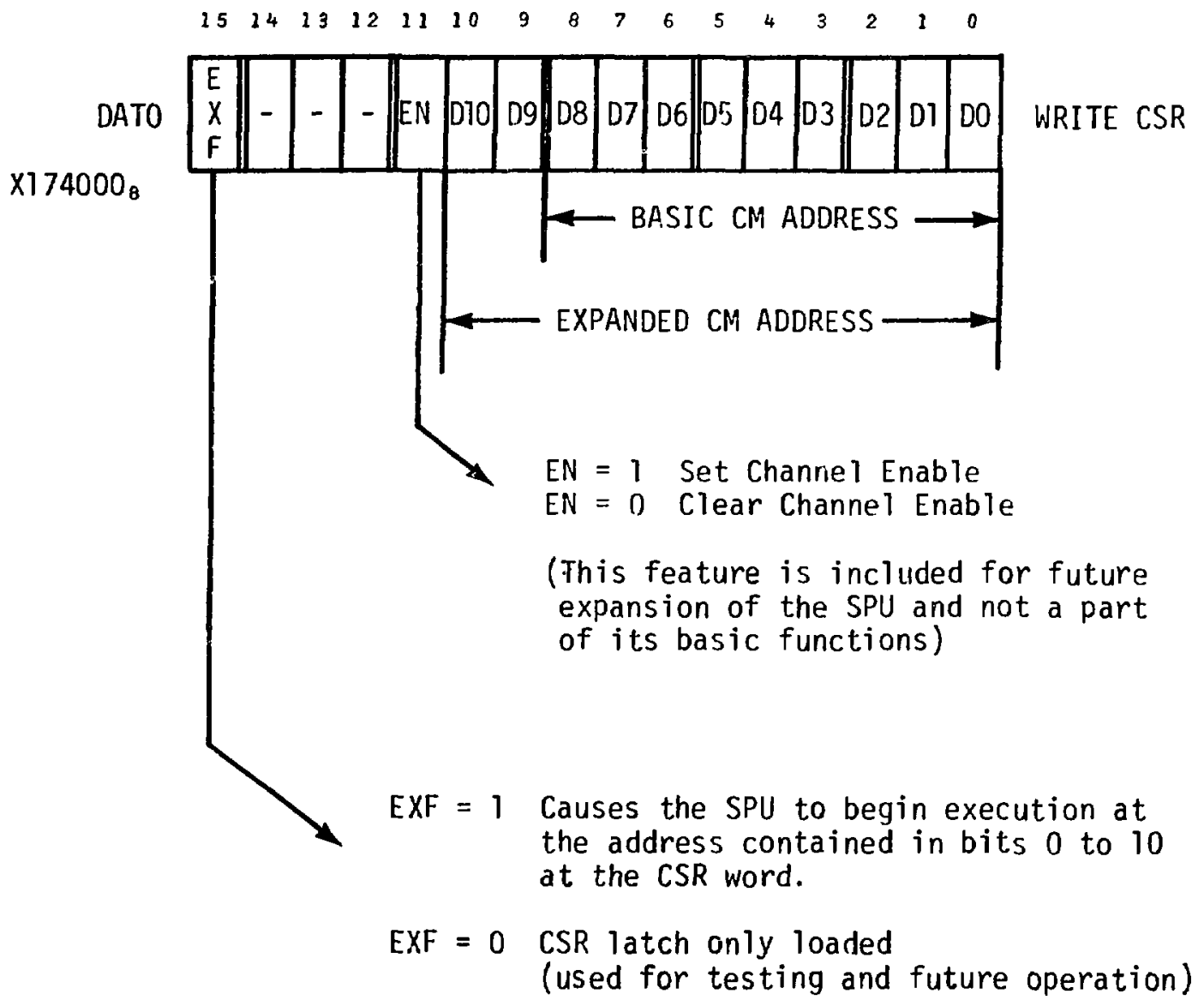




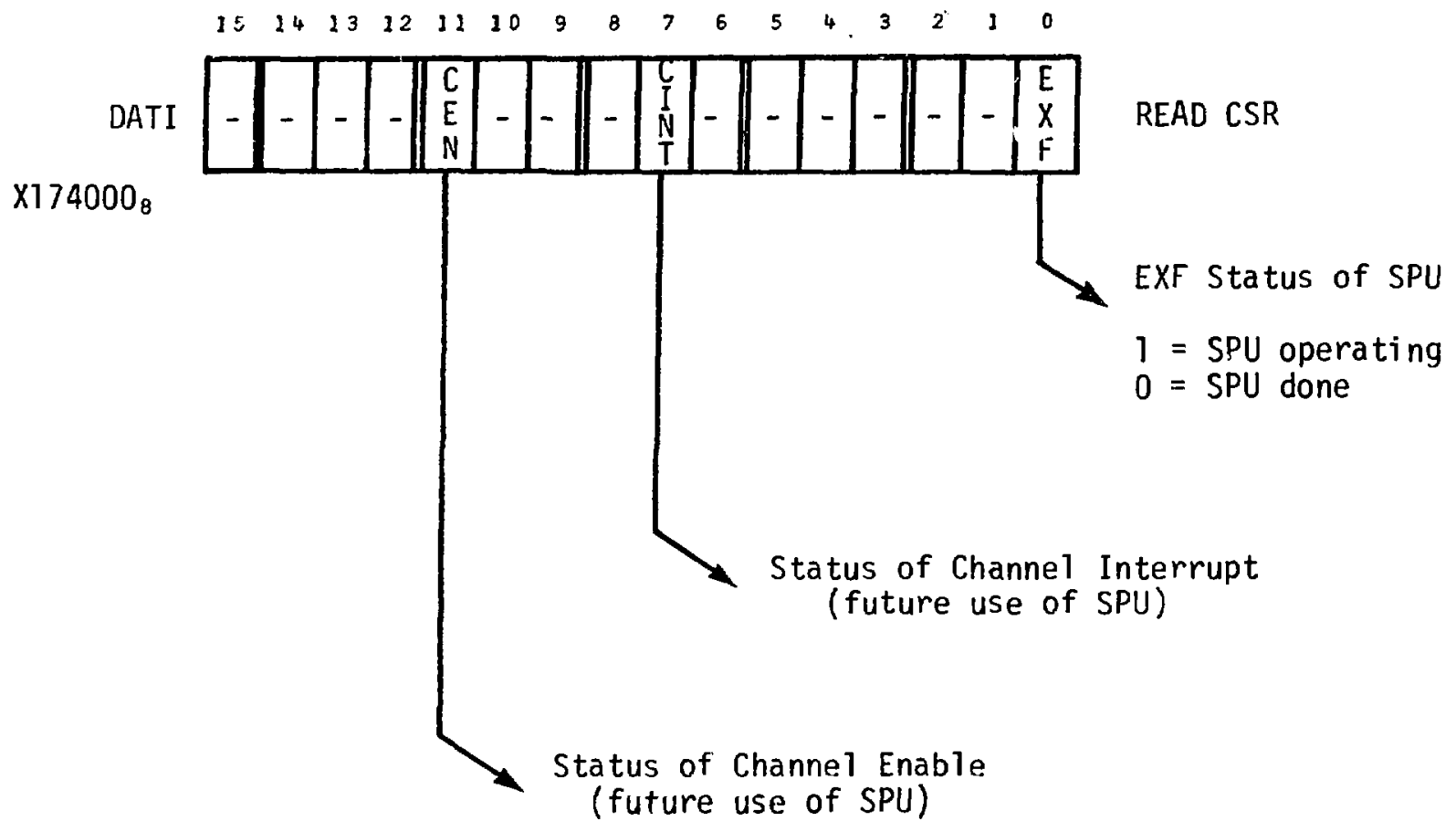

A 16-bit ALU with shift function control, look-ahead carry, and 16 input/ output signal lines is controlled from inother field of the micro-instruction. The ALU function, source and destination (one of 16 on-chip registers or the $I / 0$ pins), and direction of data transfer are controlled from this field.

Test conditions from the ALU (zero, negative, etc.) along with six special LAM signals are sent to the test condition Multiplexer previously mentioned. At each instruction time, the sequencer can test a condition that is staticized during the last cycle.

Yet another field of the micro-instruction controls the Q-Bus interface through a control function decoder. The state machine is able to request a DMA hold on the Q-Bus, execute a READ (DATI) or WRITE (DATO) cycle on the bus and transfer data to and from the interface to the ALU. Another important feature of the bus interface is the one-way parameter transfer RAM. As in a software subroutine, we must be able to transfer operating parameters to the firmware subroutine facility. This is accomplished by a $16 \times 16$ RAM which may be written from the Q-BUS and read by the SPU. The on-chip ALU registers are a reflection of this RAM, and can be used to hold similar working parameters. Note that the RAM cannot be written from the ALU, nor can it be read by the Q-Bus.

The Parameter RAM is available at address:

$$
\text { X174040 - X174076. }
$$

An 8-switch "DIP-Switch" located on the module allows the address expansion (page) bits to be set and, in addition, 16 other SPU base addresses may be selected so that a maximum of $16 \times 4$, or 64, individual SPUs can be operational in one LSI-11/2 controlled ACC. 


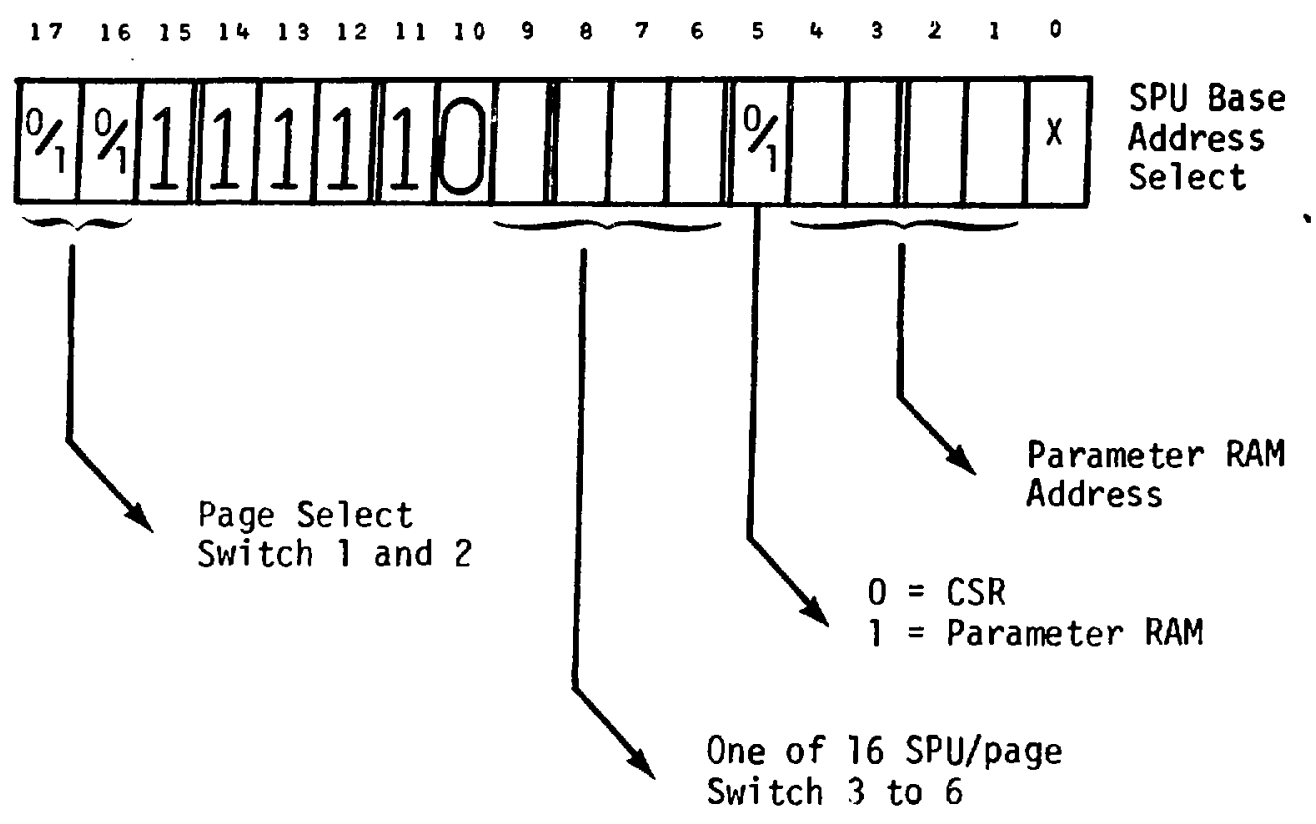

SPIJ

Base Address

174000

374000

574000

774000

$\times 174000$

$\times 174100$

$\times 174200$

$\times 174300$

$\times 174400$

$\times 174500$

$\times 174600$

$\times 174700$

$\times 175000$

$\times 175100$

$\times 175200$

$\times 175300$

$\times 175400$

$\times 175500$

$\times 175600$

$\times 175700$

Switch No.

\begin{tabular}{ll}
1 & 2 \\
\hline 0 & 0 \\
0 & 1 \\
1 & 0 \\
1 & 1
\end{tabular}

3

4

5

6

0
1
0
1

$\begin{array}{llll}0 & 0 & 0 & 0 \\ 0 & 0 & 0 & 1 \\ 0 & 0 & 1 & 0 \\ 0 & 0 & 1 & 1 \\ 0 & 1 & 0 & 0 \\ 0 & 1 & 0 & 1 \\ 0 & 1 & 1 & 0 \\ 0 & 1 & 1 & 1 \\ 1 & 0 & 0 & 0 \\ 1 & 0 & 0 & 1 \\ 1 & 0 & 1 & 0 \\ 1 & 0 & 1 & 1 \\ 1 & 1 & 0 & 0 \\ 1 & 1 & 0 & 1 \\ 1 & 1 & 1 & 0 \\ 1 & 1 & 1 & 1\end{array}$

A closed, or on switch $=1$ logic 0 ; an open, or off switch $=\operatorname{logic} 1$. 


\section{SPU Timing Measurements}

SPL-generated Q-Bus cycle timing measurements are included in the following table. The time recorded is both the SYNC assertion time on the Q-Bus and the number of SPU cycles; a measure of SPU uncertainty is important in measuring the actual speed of operation of a fast register transfer device. Also, the $R / W$ times were measured by asking the SPU to execute a $R / W$ operation a large number of times and then averaging the total time over the number executed.

\begin{tabular}{rccc} 
Source and Destination & Read & Write & R/W \\
\cline { 1 - 3 } 50 ns RAM & $225 / 6$ & $225 / 6$ & $1250 / 13$ \\
200 ns RAM & $575 / 9$ & $575 / 9$ & $2000 / 19$ \\
CAMAC (norma cycle) & $1000 / 11$ & $1000 / 11$ & $2600 / 23$ \\
CAMAC (short cycle) & $600 / 9$ & $600 / 9$ & $2000 / 19$
\end{tabular}

The first figure is the actual cycle time in ns and the second is the number of SPU cycles required to complete the cycle.

\section{Future Addi tions to the SPU}

The SPU has been designed and constructed such that options can be added should the need exist. Mention has been made of the channel controller, a 2-MHz bit serial communications-channel controller which formats information into the SOLC or Bi-Sync line protocol. A device of this nature has been foreseen but never actually implemented in an ACC system. Hooks have been left, however, should this form of communication from a remote CAMAC crate be desired (see Ref. 8). Secondly, the necessary connection points have been designated and brought to wirewrap pins such that a 100-ns $8 \times 8$ combinatorial multiplication chip could be added. This multiplier can greatly speed up the process of multiplication in the SPU, and should be immediately added when it hecomes necessary to multiply in a front-end preprocessing application.

Finally, by use of the PROM simulator connectors, an expanded control memory (up to $2 K$ words) can be added to the SPU. In all three future possible additions, a second pC board must be added making the SPU a 2-wide module.

G. The SPU PROM Simulator

The simulator is a CAMAC module designed to aid in checkout of SPU code. A se $i$ of Fairchild 35392 Static RAMs have been arranged in an array of $512 \times 40$, or five $512 \times 8$, depending upon the mode of operation. In the former case, the SPU accesses the RAMs over the 20-pin SPU address connector and receives a 40-bit micro-instruction via the 40-pin SPU data connector. In the latter case, a system controller, via the Dataway holding the simulator module, can address and access the RAMs for load-up of SPU code.

The simulator is a 1-wide CAMAC module requiring $1.3 \mathrm{~A}$ from the $+5 \mathrm{~V}$ rail. 


\section{CAMAC Command}

Subaddress A0

WT1 (F16) Write Memory Address (W12-W1)

Register and set CAMAC Access Mode

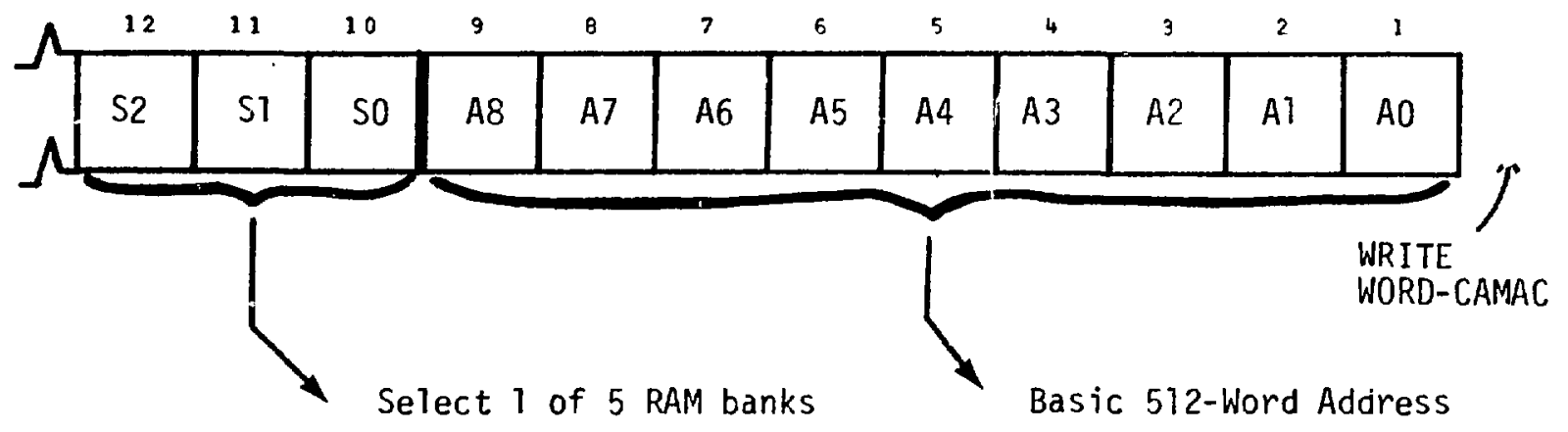

The select code references the following micro-instruction fields:

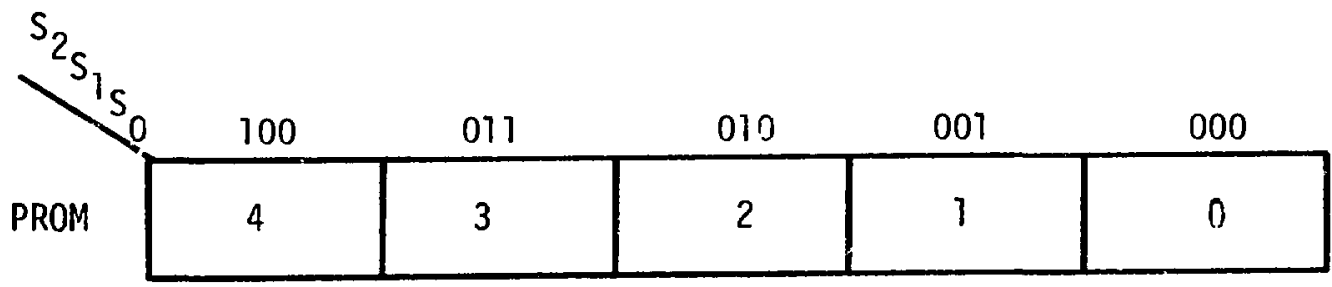

WRITE bits W9-W7 then select which word within the selected block will be accessed.

RDI (F0) Read the contents of the MAR (R12-R1) and clear the CAMAC Access Mode.

Subaddress A1

WT1

Write Memory at MAR, when complete MAR + MAR+1.

During BUSY, assert Test Inhibit to SPU.

W8-W1 are transferred to the selected address at the selected bank.

RDI Read Memory at MAR, when complete MAR $\leftarrow$ MAR+1.

During BUSY, assert Test Inhibit to SPU.

R8-R1 contain the contents of the selected address at the selected bank. 
The above four CAMAC instructions only will return $x$ to the Crate Controller. The simulator responds to Z.S2 by clearing MAR to zero and clears CAMAC access mode.

Dperating Sequence

The user should follow the recommended procedure for loading and testing the simulator memory or some data may be invalid.
WTI·AO
Set MAR and set CAMAC Access Mode.
WTI.AI Write data.
WTI-AO Set MAR to beginning.
RDI.Al Read and check.
RD1-A0 Read MAR to check and clear CAMAC Access Mode.
The simulator is then ready for access by the SPU. Note that the SPU must run with a 1-MHz crystal in place of the 7-MHz crystal during operation with the PROM simulator. All PROMs must be removed during simulation.

\section{H. Minimum Controlier}

A 2-wide module has been constructed to take the place of a "normal" crate controller located in crate stations 24 and 25 . The minimum controller provides pull-ups for all Dataway and Auxiliary Controller Bus signal lines, and a station number $(N)$ decoder for the Auxiliary Controller Bus (ACB). LAM signals are routed to the ACB connector which is located at the rear of the minimum controller.

The primary use of the minimum controller is in stand-alone operation of a crate with ACC, or expansion of an ACC system to another crate by use of a single control port (see Fig. 3, Section I). 


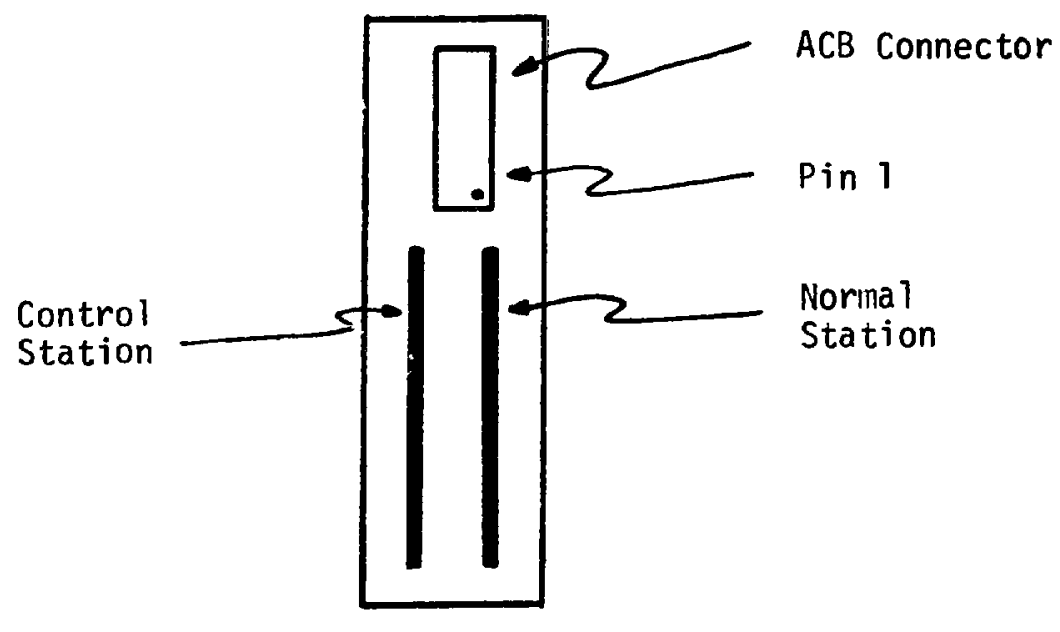

Rear View of Module

\section{ACC SOFTWARE} sys tem:

Three questions are usually asked when people consider using the ACC in a

1. How do I program the primary processor?

2. How do I transfer code to the ACC?

3. How do I program the SPU?

The first question may be answered immediately in our case--use any PDP-11 computer to create, edit, assemble (or compile), and link code. There are only a few differences in the instruction sets between the various PDP family machines", and these may be handled by macros or DATA statements.

The second question is somewhat more involved. The code transfer medium can be the following:

1. Down-line load through a branch highway, serial highway (or other compatible interface) through the Dataway Access Port of the ACC. The user must convert his .LDA files to an appropriate data stream to load memory in the ACC.

2. Use a data communications port on the development computer to the terminal port on the LSI-11/2 board. Software must be written on both the development computer and the LSI-11/2 (resides in boot PR.OM) to handle this transfer technique.

3. The cassette tape unit mentioned in Section I can be loaded with linked code on some other computer system and then read by the LSI-11/2 boot. Software for this unit has not yet been developed, but will be reported on in a later paper dealing with beam line control using the ACC.

The third question is yet more difficult to answer and will be postponed to a separate section in this report (see Section IIIB). 


\section{A. The Basic Debug Code}

A technique for working with an ACC through a development computer system was used at the Daresbury Laboratory during the engineering of the first ACC. A similar debug technique was put together by J. Potter of the MP-I staff during the engineering of the LAMPF ACC. The computer uses the RT-11 disk operating system and has a dual floppy disk as a mass storage medium, together with a fully implemented CAMAC crate with parallel branch highway driver. Using the derivative of BASIC for special I/0, CALLBASIC, an ACC Debugger was written (called ACBUG) to allow the ACC user to easily work with the controller and test code during a development session. The load-up and execution of ACBUG is shown in Fig. 5. Note that the position of the Dataway Access Port must be input to the code. Communications with the ACC is via the Branch Driver, CCA-2\# or SCC-L2 Crate Controller and the Access Port.

RUN BASCAM

BASIC VOIB-02

*

USER FNS LOADED

READY

OLD"ACBUG"

READY

RUN

ACBUG 21-SEP-78 BASIC V01B-02

ACC DATAWAY ACCESS PORT SLOT $=? \underline{8}$

$\begin{array}{llll}\text { *SELECT........ RUN } & \text { ODT } & \text { BOOT } & \text { INIT } \\ \text { RDSTAT } & \text { LDMEM } & \text { DMPMEM } & \text { TSTMEM } \\ \text { *COMMAND........ DXRAM } & \text { RAMDX } & & \end{array}$

?

Fig. 5. Debugger commands.

User Input

The meaning of the ACBUG commands is noted below. A sample session with ACBUG is shown in Fig. 6 .

FuN Enable RUN and issue a RESET to rorce the LSI- $11 / 2$ to $173000_{8}$. This command should be executed when leaving ODT.

ODT Forces the HALT line to be asserted and transfers control to ODT microcode in the LSI-11/2. If a terminal is connected to the LSI-11/2 port, one can use ODT directly into the $11 / 2$.

\#LEEP has ten modified $A-1$ controllers (CCA-1 1/2) that are compatible with ACCs, in addition to five CCA-2 controllers. 


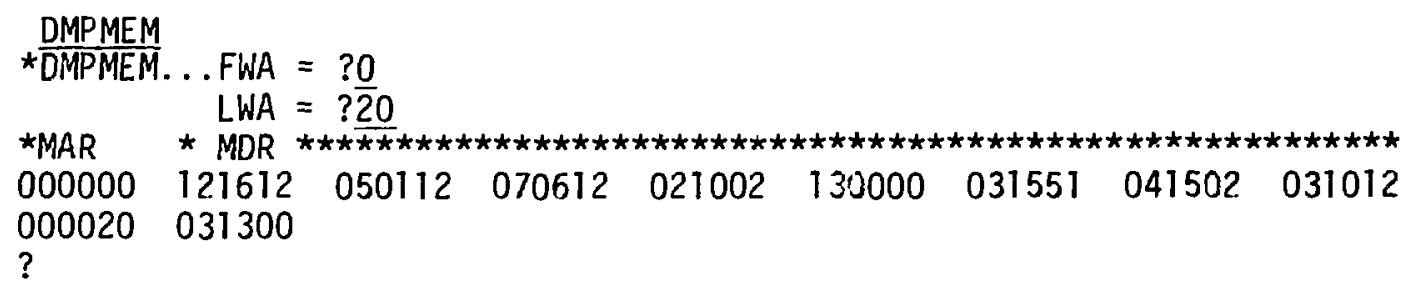

Fig. 6. Sample ACBUG session. 
BOOT Forces a RESET and transfer to $173000_{B}$ for bootstrap purposès.

INIT Generates a Q-Bus INIT signal to reset all devices connected, with the exception of the $11 / 2$.

RDSTAT Reads the Status Register in the Access Port and prints the result.

LDMEM A line-by-line load of memory from a starting address given.

DMPMEM Reads a given block of memory and prints the result.

TSTMEM Tests memory over a given address block, zeroes, ones, alternating bit pattern.

DXRAM Transfer an LDA format file on disk to memory.

RAMDX Transfer a given address block to disk in LDA format.

\section{B. SPU Microcode}

The SPU Control Word, derived from Control Memory (CM) is 40-bits long and provides the user with a detailed level of facility control not available on any single-chip microprocessor or microcomputer. (The LSI-11/2 is microcoded, but the user cannot generally have access to this microcode.) The reader is referred back to Fig. 4, the block diagram of the finite state machinet and Q-Bus interface making up the SPU, for the discussion that follows. In addition, Fig. 7 in this section contains the $\mathrm{CM}$ field formats and a mnemonic list of the field definitions useful when coding the machine. Finally, Fig. 8 shows the interim coding sheet and is included at the rear of this section. This rather basic coding form indicates how code is written for the SPU in order that, lacking a cross-microassembler, one can keep track of all the bits!

A future recormendation is to acquire a cross-microassembler* that will operate on a 16-bit minicomputer system. Integral to that, a fuse-Tink PROM programmer** CAMAC module should be installed with the microcomputer development system such that PROMs can be programmed directly from files generated by the cross-microassembler. Alternatively, these files can be written to the PROM simulator for test and debug prior to programming PROMs.

Testing and initial programming of the SPU was accomplished by a hand programmer and is too prone to errors to be considered the way to proceed in the future (a fresh CM array contains $20 \mathrm{~K}$ bits waiting to be programmed).

When microcoding the SPU, a mental picture of the entire facility must be kept at hand. The architecture of the machine is both vertical and horizontal, which means that some in fields are shared by two or more functions, some are directly (unary) assigned, and same fields are coded.

fUsing AMD-2910 Sequencer and four AMD-2901A ALUs.

*Available from MICRO-TEC, Sunnyvale, CA., and installed in December 1978. **Design received from CERN LINAC group in October 1978. 


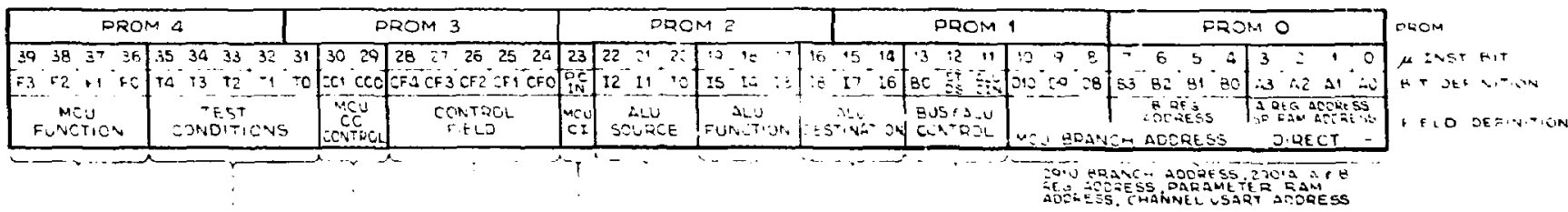

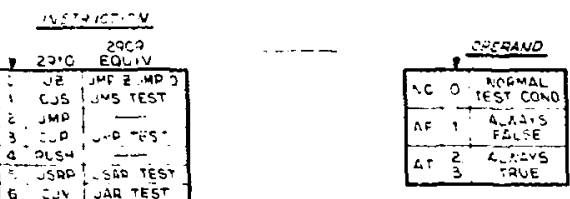

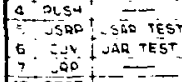

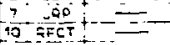

19. FECT OCNTCONT

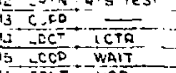

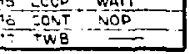

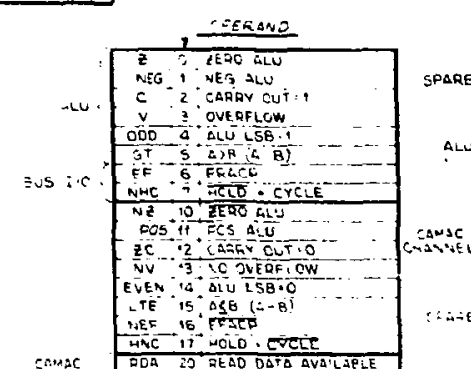

SPARE

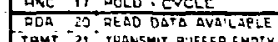

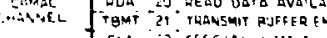

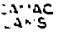

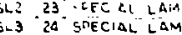

$5 L 4$ - is. $50 E-5$ L

$=26 \pi_{37}$ SOECLAL LAM 6 .

ONA 35 . PATA WOT AVALABLE

- EF,

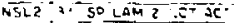

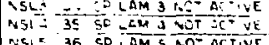

NSL6 37 50

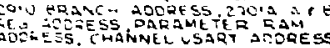

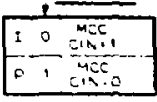

nore

QEFER TO ZTO LESICN SFECSFCR DETAILS
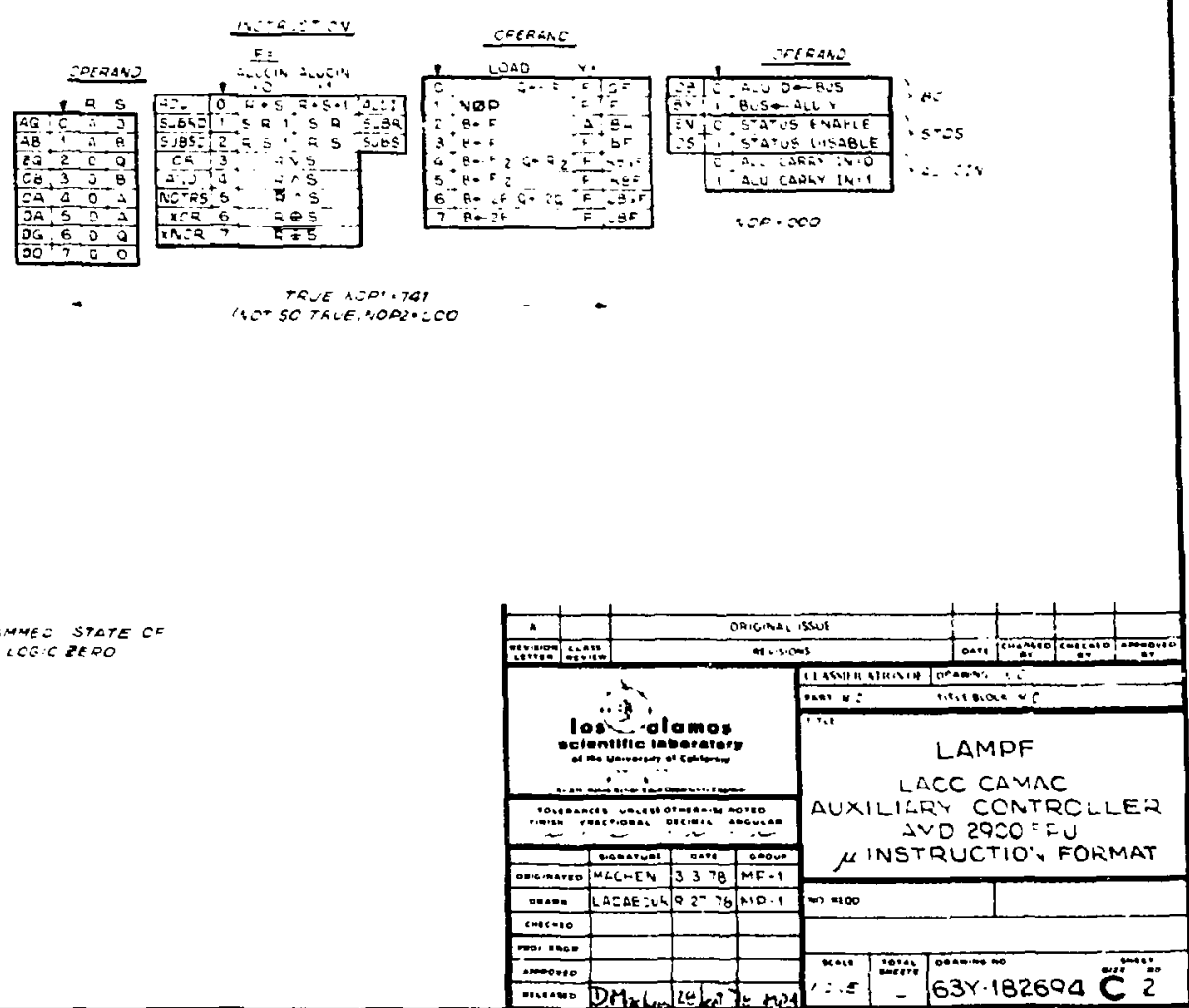

Fig. 7. Special processor unit microcode format. 


\begin{tabular}{lll} 
Coded Fields & Width & Designation \\
\hline 4-bits & Sequencer Function \\
5 -bits & Test Condition \\
5 bits & Control Field \\
9 bits & ALU Source, Function and Destination
\end{tabular}

Direct Assignments

$\begin{array}{ll}2 \text { bits } & \text { Sequencer Condition Control } \\ 1 \text { bit } & \text { Sequencer Carry Control } \\ 1 \text { bit } & \text { Bus Transfer Direction } \\ 1 \text { bit } & \text { ALU Carry Control } \\ 1 \text { bit } & \text { Status Latch Enable } \\ 3 \text { bits } & \text { D-Input, Sequencer (D10-D8) }\end{array}$

Shared Fields

$$
\begin{array}{ll}
\text { 4-bits } & \text { ALU A Register, Parameter RAM and } \\
& \text { Sequencer (D3-DO) } \\
\text { 4-bits } & \text { ALU B Register and Sequencer (D7-D4) }
\end{array}
$$

The sequencer or Micro-Control Unit (MCU) is capabie of generating a 12-bit address; we are using only 11 bits in the SPU design. On-board memory accounts for 9 of the 11 possible.

In the discussion to follow, it will be assumed that the reader has a reasonable understanding of the state machine and the bus interface logic of the SPU. The state machine will be considered from the point of view of its microcode, where possible, and not from the point of view of the hardware.

Referring to Fig. 7, a line of microcode might look like:

$$
\text { CJP } P \text { NC SHO AB OR B } 2 F \quad 001 \text { B2 } A 4 \text {, }
$$

which would select the ALU registers $B=B 2$ and $A=A 4$, logically $O R$ the contents of $B 2$ and $A 4$, left shift the result with zero fill and store the results in B2. The ALU Y outputs are disabled, the ALU carry-in is set to logic 1, and the status latch is enabled. This instruction wi 11 be executed once, and the condition of the ALU D:Itput will be tested; execution will proceed at the address in CM formed by the concatenation of $(B 2, A 4)$ if the ALU output is positive, or contirue with the next instruction if the output is otherwise.

The reader must appreciate the somewhat subtle effect of a Pipe-Line Register (PLR) between a CM and the various control word-field destinations. The PLR is clocked on the rising edge of the SPU clock, allowing CM to settle to a new address during the clock period. The MCU is generating this $\mathrm{CM}$ address (for the operation to follow the one currently held in the PLR) by special stack operations, selecting other sources or by incrementing the current address (and holding $i t$ ), or passing the current address to a hold, dependent upon the state of the test condition inputs/MCU carry-in. The source of the next address can be from several different areas (CTR/REG, Direct, and Zero) depending upon the function code, as discussed below. 
MCU Function Codes

The instruction field in the high-byte $6 \dot{r}$ PROM 4 (F3-FO) is used to instruct the 2910 to select a next address from one of several sources. Generally, a Test Condition modifier will be used with most micro-instructions, and is selected by the next control field (T4-TO) and the condition control bits (CCl,CCO). Either normal condition testing (selected by T4-TO), Always True or Always False, allow complete control of the MCU condition control inputs.

Finally, the microprocessor on the MCU can be directly controlled by the carryin to the microprocessor incrementer (the bit PCIN in PROM 2).

\begin{tabular}{|c|c|c|c|c|c|c|c|c|}
\hline$F_{3}-F_{0}$ & MNEMDNIC & NAME & $\begin{array}{l}\text { REG/ } \\
\text { CNTR } \\
\text { CON- } \\
\text { TENTS }\end{array}$ & \multicolumn{2}{|c|}{ FAIL } & \multicolumn{2}{|c|}{ PASS } & $\begin{array}{l}\text { REG/ } \\
\text { CNTR }\end{array}$ \\
\hline 0 & $\mathrm{JZ}$ & JUMP ZERO & $x$ & 0 & CLEAR & 0 & CLEAR & HOLD \\
\hline 1 & CJS & COND JSB PL & $x$ & $\mathrm{PC}$ & HOLD & $\mathrm{D}$ & PUSH & HOLD \\
\hline 2 & $J M P$ & JUMP & $x$ & D & HOLD & $\mathrm{D}$ & HOLD & HOLD \\
\hline 3 & CJP & COND JUMP PL & $x$ & $P C$ & HOLD & $\mathrm{D}$ & HOLD & HOLD \\
\hline 4 & PUSH & PUSH/COND LD CNTR & $x$ & $\mathrm{PC}$ & PUSH & PC & PUSH & * \\
\hline 5 & JSRP & COND JSB R/PL & $x$ & $R$ & PUSH & $D$ & PUSH & HOLD \\
\hline 6 & CIV & COND JUMP VECTOR & $x$ & $\mathrm{PC}$ & HOLD & D & HOLD & HOLD \\
\hline 7 & $J R P$ & COND JUMP R/PL & $x$ & $R$ & HOLD & D & HOLD & HOLD \\
\hline \multirow[t]{2}{*}{10} & \multirow[t]{2}{*}{ RFCT } & \multirow[t]{2}{*}{ REPEAT LOOP, CNTR $\neq 0$} & 70 & $\mathrm{~F}$ & HOLD & $F$ & HOLD & DEC \\
\hline & & & $=0$ & PC & POP & $\mathrm{PC}$ & POP & HOLD \\
\hline \multirow{2}{*}{11} & \multirow{2}{*}{ RPCT } & \multirow{2}{*}{ REPEAT PL, CNTR $\neq 0$} & $\neq 0$ & $D$ & HOLD & $D$ & HOLD & DEC \\
\hline & & & $=0$ & PC & HOLD & $\mathrm{PC}$ & HOLD & HOLD \\
\hline 12 & CRTN & COND RTN & $x$ & PC & HOLD & $F$ & POP & HOLD \\
\hline 13 & CJPP & COND JUMP PL \& POP & $x$ & $P C$ & HOLD & $D$ & POP & HOLD \\
\hline 14 & LDCT & LU CNTR \& CONTINUE & $x$ & PC & HOLD & PC & HOLD & LOAD \\
\hline 15 & LOOP & TEST END LOOP & $x$ & $\mathrm{~F}$ & HOLD & $P C$ & POP & HOLD \\
\hline 16 & CONT & CONTINUE & $x$ & $\mathrm{PC}$ & HOLD & PC & HOLD & HOLD \\
\hline \multirow[t]{2}{*}{17} & \multirow[t]{2}{*}{ TWB } & \multirow{2}{*}{ THREE-WAY BRANCH } & $\neq 0$ & $F$ & HOLD & $P C$ & $\overline{P O P}$ & DEC \\
\hline & & & $=0$ & $D$ & POP & $P C$ & POP & HOLD \\
\hline
\end{tabular}

\#If condition fails, then hold register. If condition passes, then load register with $(D, B, A)$.

The table (reproduced and modified from that available in the 2910 literature) details what control each MCU function has over the on-chip facilities. A brief description of each function code follows: 
Command 0 - JZ - Jump Zero is used to jump to the beginning of CM. When used at the end of a program (or when a reset is applied), the command unconditionally sets the next address to be zero. To remain at location zero while testing a selected condition, $P C I N=1$.

Command 1 - CJS - Conditional Jump to Subroutine via the address in $(D, B, A)$. If the condition is true, the next address in sequence is pushed onto the stack and the program jumps to the address specified in $(D, B, A)$. For a false condition, the next address in sequence is selected.

Command 2 - JMP - Unconditional jump to address in $(D, B, A)$.

Command 3 - CJP - Conditional instruction which, if true, selects $(0, B, A)$ as the next address. Very sirilar to Command 1 except that the next address in sequence is not pushed onto the stack.

Command 4 - PUSH - Conditional instruction which pushes the next address in sequence onto the stack and if the condition is true, the counter is loaded from the PLR branch address field. If false, the counter is not loaded.

Command 5 - JSRP - Conditional Jump to Subroutine via PLR address or register address. A push onto the stack is always performed, and if the condition is true, the register contents will be used as the next address. If false, the PLR branch address field will be used as the next address. The register must have been loaded with the correct value for the subroutine address by the instruction prior to the Conditional Jump to Subroutine.

Command 6 - CJV - Conditional Jump Vector instruction is used to load the SPU with the address from the CSR when the External Function bit (bit 15) is also set to logic 1. This instruction is placed at address 0 , and until an External Function is received, the condition will remain false. If, at the same time, $P C I N=1$, the address will not be incremented and the program will hang at address 0 . When an External Function occurs, the condition will become true and the program will go to the address supplied by the CSR address field. (See Section IIF on SPU hardware design and operation.)

Command 7 - JRP - Conditional Jump instruction via the contents of the Register Counter, or the PLR branch address field. This is similar to Conmand 5 except that no push onto the stack occurs.

Command 10 - RFCT - Repeat Loop, Councer $\neq$ Zero instruction assumes that a count has been loaded into the Register/Counter. If the count is not zero, the count is decremented by 1 and the address of the next micro-instructrion taken from the stack. When the count is zero, control passes to the rext sequential micro-instruction and the stack is pop'ed.

Command 11 - RPCT - Repeat Pipeline Register, Counter $\neq$ Zero instruction is similar to Command 8 except that the branch address in the event of the count $\neq$ 0 comes from the pipeline register rather than the stack. Also, a pop of the stack is not performed.

Command 12 - CRTN - Conditional Return from Subroutine instruction will return to the main program by completing a pop on the stack if the condition is true. If false, the next micro-instruction in sequence in the subroutine will be performed.

Command 13 - CJPP - Conditional Jump Pipeline Register and Pop stack instruction. If the condition is false, the program will sequence onto the next micro-instruction. If true, the program will jump to the address supplied by the PLR branch address field, and the stack will be pop'ed.

Command 14 - LDCT - Load Counter and Continue instruction allows the counter register to be loaded with an 11-bit number. 
Command 15 - LOOP - Conditional Test End of Loop instruction causes the next instruction in sequence to be executed if the condition is true. If the condition is false, the microprogram will loop back via the address held on the top of the stack file. This instruction would normally be placed at the end of a loop.

Command 16 - CONT - Continue instruction which causes the microprogram to increment to the next micro-instruction in sequence. A NOP.

Command 17 - TWB - Three Way Branch instruction which assumes that the register/counter has been preloaded and a branch address has been pushed into the stack. The instruction decrements the count and branches to the address given by the stack as long as the counter is greater than zero. When the counter $=0$, the next address is selected from the PLR branch address field. However, if at any time the condition code becomes true, the microprogram counter, (i.e., pc), will be the new address selected. The stack is automatically pop'ed whenever the count reaches zero or the conditional test becomes true.

Test Conditions

The selectable Test Conditions (TC) relate to fixed terms (true and false for each), ALU generated terms, or bus I/O generated terms. TC(20), TC(21), and TC(30), TC (37) are reserved for future possible use with a communications channel controller. TC $(0)-T C(5)$ and TC(10)-TC(15) are ALU terms and self explanatory, while $\mathrm{TC}(6), \mathrm{TC}(7)$ and $\mathrm{TC}(16), \mathrm{TC}(17)$ require more detail. The latter conditions refer to the External Function control bit in the CSR and the DMA hold/ cycle-in-progress signals respectively, from the bus I/0. By using these TCs, all possible states of the bus, of concern to the SPU, may be tested.

TC(23)-TC(27) and TC(33)-TC(37) allow the state of the six Special LAMs from the ACC Control Port to be tested. Finally, the STDS control bit (in PROM 1) controls the enable/disable state of the TC staticizer. TCs are latched on the rising edge of the SPU clock (the beginning of a cycle); the TCs may be held from the last cycle by letting STDS $=1$ in the current and subsequent cycles.

\section{Condition Control}

The user is able to determine the effect of the test conditions on the MCU through bits 30 and 29 of the micro-instruction; $\mathrm{CCl}$ and $\mathrm{CCO}$ can take on values that define either "Normal Conditions" - NC (0), "Always True" - AT (1), or "Always False" - AF (2 or 3). This field is useful when used with the conditional MCU instructions, as shown in the MCU table of instructions.

\section{Control Field}

The Control Field provides the SPU with control over all bus I/0 options, shift multiplexer function, and future options.

The shift function controls (CF(14)-CF(17)) have been repeated every four decoded commands, but are unique for commands 14-17. It is possible then to execute a shift function while performing some other control.

CF(i)

NOP $\quad C F(0)$

DMA
Function

- No control action; ALU shift logic set for shift logical with zero fill.

- Request DMA hold on ACC bus (tested with TC(7), NHC or TC(17) HNC). Shift logic: Logical, 1 fill. 
CF(i)

Furiction

\begin{tabular}{|c|c|c|}
\hline CDE & $C F(2)$ & $\begin{array}{l}\text { - Clear DMA hold and External Function bit in CSR. } \\
\text { Shift logic: Rotate. }\end{array}$ \\
\hline LBA & $C F(3)$ & $\begin{array}{l}\text { - Load the Bus Address Register from ALU outputs; } \\
\text { Shift logic: Arithmetic. }\end{array}$ \\
\hline LBD & $\mathrm{CF}(4)$ & $\begin{array}{l}\text { - Load the Bus Data Register from ALU outputs; } \\
\text { Shift logic: Logical, o fill. }\end{array}$ \\
\hline $\mathrm{RC}$ & $\mathrm{CF}(5)$ & $\begin{array}{l}\text { - Request Read cycle on ACC bus; use contents of BAR } \\
\text { as address, data to BDR. } \\
\text { Shift logic: Logical, i fill. }\end{array}$ \\
\hline$W C$ & $C F(6)$ & $\begin{array}{l}\text { - Request Write cycle on ACC bus; use contents of BAR } \\
\text { as address, data from BDR. } \\
\text { Shift logic: Rotate. }\end{array}$ \\
\hline RRAM & $\mathrm{CF}(7)$ & $\begin{array}{l}\text { - Read contents of SPU parameter memory, at address } \\
\text { in A-Field, into ALU direct inputs }(B C=0) \text {. } \\
\text { Shift logic: Arithmetic. }\end{array}$ \\
\hline RBD & $\mathrm{CF}(10)$ & $\begin{array}{l}\text { - Read contents of BDR into ALU direct inputs }(B C=0) \text {. } \\
\text { Shift logic: Logical, } 0 \text { fill. }\end{array}$ \\
\hline- & $C F(11)$ & - Spare; may be used for multiplier option. \\
\hline- & $\mathrm{CF}(12)$ & - Spare; may be used for multiplier option. \\
\hline- & $\operatorname{CF}(13)$ & - Spare; may be used for multiplier option. \\
\hline SHO & $\operatorname{CF}(14)$ & - Logical shift ALU, zero fill. \\
\hline SHI & $\operatorname{CF}(15)$ & - Logical shift ALU, 1 fill. \\
\hline SR & $C F(16)$ & - Rotate ALU. \\
\hline SA & $\operatorname{CF}(17)$ & - Arithmeti: shift ALU. \\
\hline
\end{tabular}

ALU Instruction Field

$\left(I_{2}, I_{1}, I_{0}\right)$ ALU source operand. $A, B, D, Q$, and zero refer to selected registers within the ALU. $A$ and $B$ are selected by the A-field and B-field of the Control Word, and refer to a multi-port $16 \times 16$ RAM in the ALU. $D$ is the ALU Direct inputs, while $Q$ is an additional working register within the ALU. Zero is truly a 16-bit, word containing zeros.

$\left(I_{5}, I_{4}, I_{3}\right.$ ) A.LU operation code (see Fig. 7 for list of possible arithmetic and logical operations).

$\left(I_{8}, I_{7}, I_{6}\right) \quad$ ALU Destination operand and output control. Register modification, storage, and shift-logic direction is controlled by this field. 
Other Micro-instruction Fields

BC Control for SPU bus-to-ALU Output or D-Input.

$$
\begin{aligned}
& 0=\text { ALUD }+ \text { BUS } \\
& 1=\text { BUS + ALUY }
\end{aligned}
$$

ALUCIN ALU carry control for arithmetic operations.

$$
\begin{aligned}
& 0=\text { Carry in }=0 \\
& 1=\text { Carry in }=1
\end{aligned}
$$

STDS Test condition latch disable. This control allows one to hold the status of some past operation that generated a set of test condition terms. $D S=1$ disables the latch.

The use of the D, A, and B- Fields have been explained in the contest of specific micro-instructions 1 isted above.

PCIN Controls the state of the MCU carry-in.

$$
\begin{aligned}
& \text { I: } \quad \text { PCIN }=0=\text { increment address } \\
& \text { P: } \quad \text { PCIN }=1=\text { pass address }
\end{aligned}
$$

Some Notes

Location 0 of Control Memory must have the following instruction:

CJV EF NC NOP $P$ NOP $000 \quad \ldots$

while a RESET or end-of-code instruction is:

JZ NOP NC CDE P NOP 000 -- .

The ALU and $(D, A, B)$ fields could contain other instruction, as needed. Note the use of an MCU Address Pass Command in both of the above instructions. Failure to follow this convention will cause address 1 to be executed in error. 


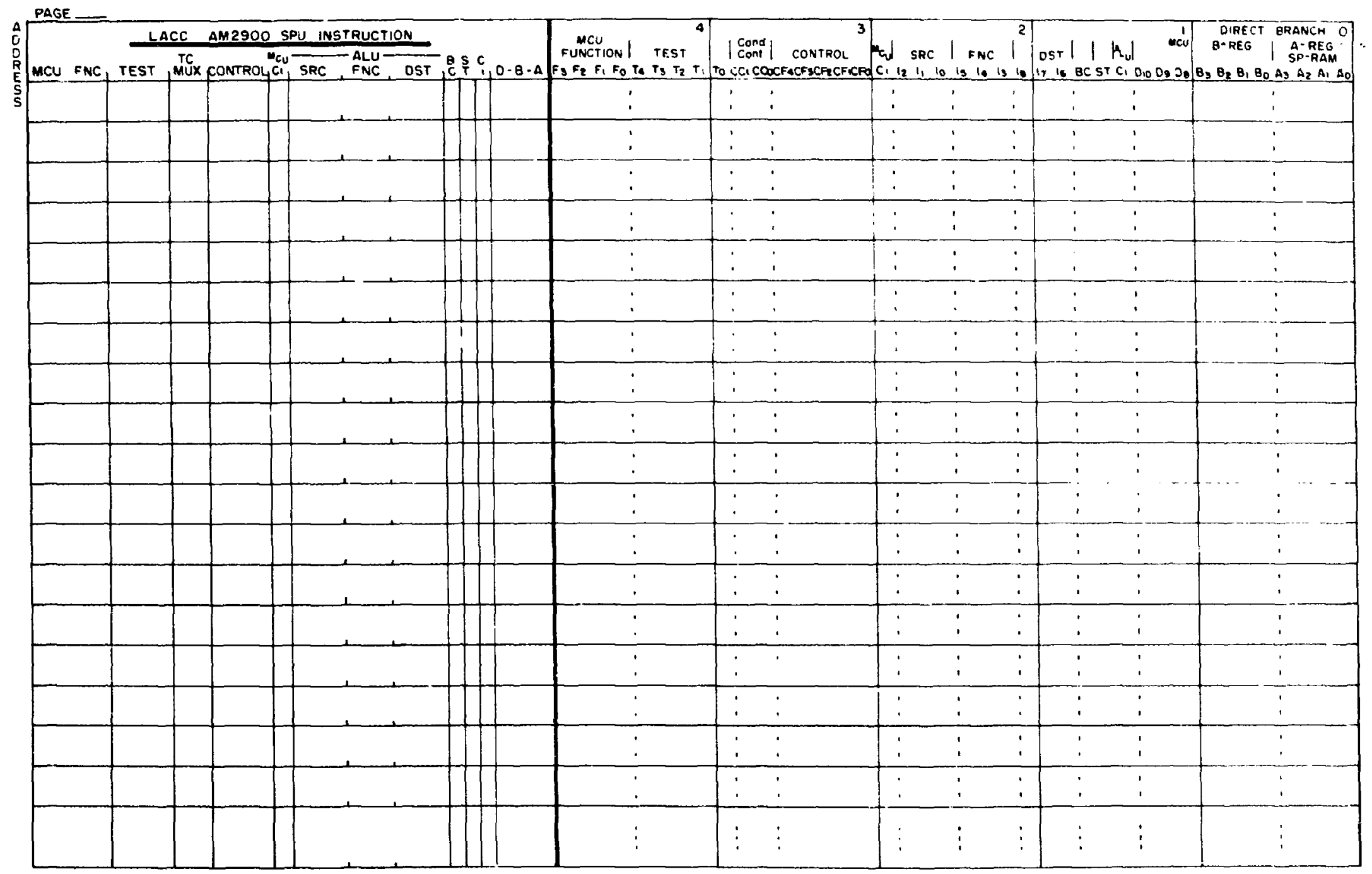

Fig. 8. Interim coding sheet. 
The following two technical notes were generated at the time the beginning ideas of The Auxiliary Controller were being formulated. I feel it is useful to include them in this report, as they bear directly on the LAMPF ACC. Only the communications controller has not been cast in hardware, although a LSI circuit* has been found that will do the job. The concept of multiple high-speed firmware subroutine facilities has been established in both bench-mark and field testing and seems to be generating a great deal of interest in the experimental physics area at LAMPF.

ॠCOM5025 from SMC Microsystems Corporation, 35 Marcus B1vd., Hauppauge, NY 11787; $\$ 24$ from Century Electronics. 


\section{CAMAC AUXILIARY CONTROLLER CONCEPTS ${ }^{+}$}

Recent discussions, prompted by the probable need for fast in-crate ADC or TDC processing at Daresbury Laboritory, have generated some interesting ideas in the area of Auxiliary Crate Controllers (ACC). This note begins an effort to tie these ideas together, with the desired outcome being a workable design approach towards a powerful in-crate processing system.

Primary Design Aim

Distribute processing tasks remotely so that:

1. System computer overhead is reduced, and

2. The data rate capabilities of the CAMAC dataway can be realized, if needed.

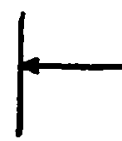

System Contrcller $\longrightarrow$ Remote CAMAC Instrumentation

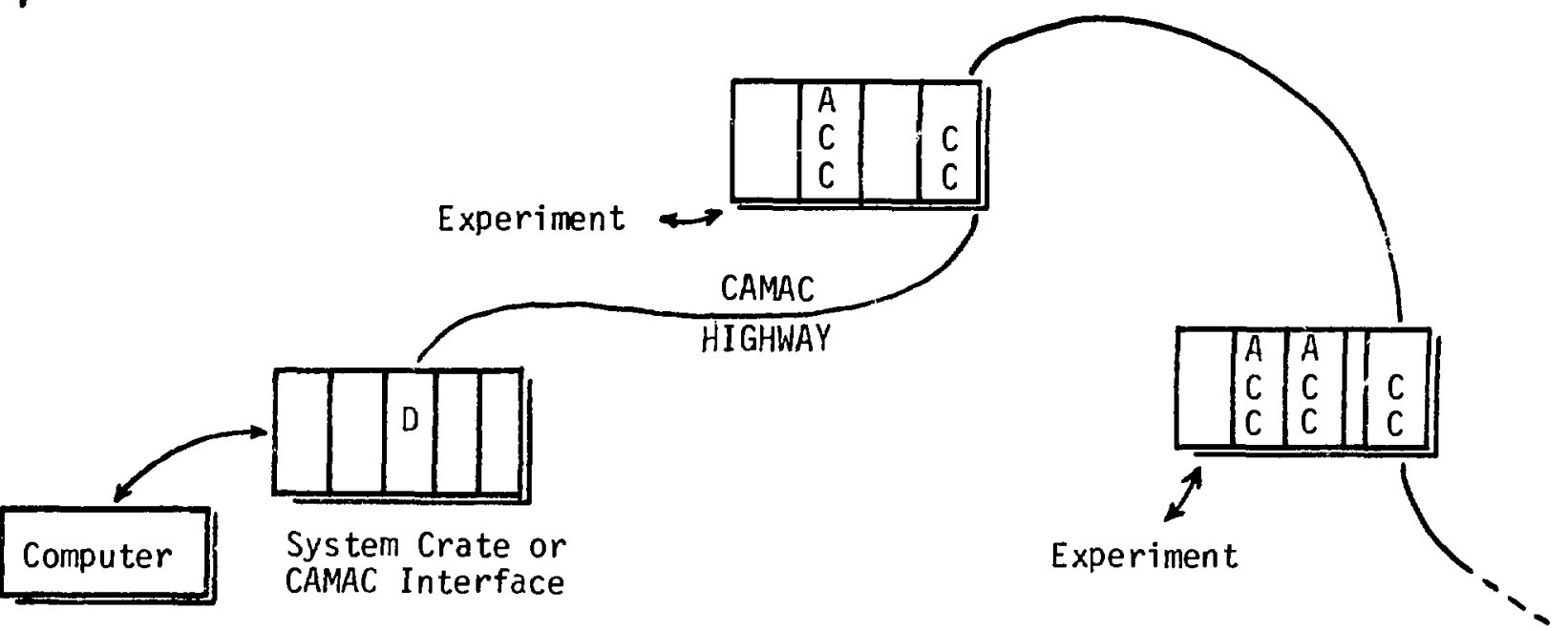

The CAMAC dataway can be effectively shared* by the CC (conventional Crate Controller) and one or more ACCs so that processing tasks can be placed within the crate. This arrangement is a logical extension of data-link communications to satellite mini computer-driven CAMAC crates, but takes advantage of the powerful microprocessor technology available to us currently.

What, then, does the ACC look like and how can it be arranged? A further question is, how can it best communicate with the system computer--other than via the CAMAC Highway (parallet or seriat)?

ACC

- One can be safe in saying that the ACC must be able to cope with normal transfers and Request/Grant or ACL priority arbitration for dataway control.

- Further, the ACC should be able to execute a fast CAMAC cycle ( $2200 \mathrm{~ns}$ ), and respond, when addressed, to some specified $C \subset$-generated CAMAC functions.

†Daresbury Laboratory Note CSE 3-76, D. R. Machen.

*DOE/EV-0007 "Multiple Controllers in a CAMAC Crate. 
- The ACC needs to have both RAM and PROM store available, but not necessarily on the same physical board.

- The ACC can be implemented with either a single-chip mirroprocessor (slower) or a bit-sliced microprogramming facility (faster).

\section{Communications with System Computer}

- B. ACC can utilize conventional LAM signaling to indicate some need to communicate with the System Computer:

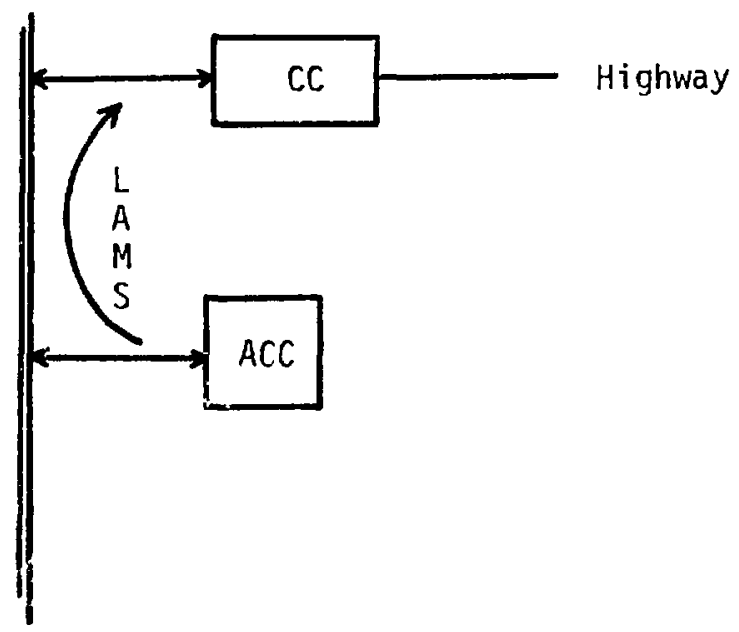

Dataway

- However, the ACC is processor-based and, therefore, will have associated with it an internal bus. Communication between the ACC and its internal elements (store, for example) can either pass along this bus or (through other dataway time-sharing techniques) alonq the dataway.

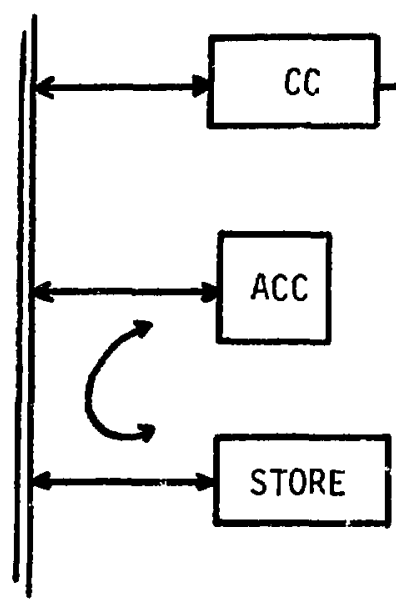

Dataway
Highway

OR

A logical extension of these ideas involves alternative communication paths to the sys tem computer. 
- As the ACC is able to exercise control over the dataway, one can install CAMAC data link (Daresbury or LAMPF-style data link) modules in the crate and operate the distributed system much like a satellite mini configuration.

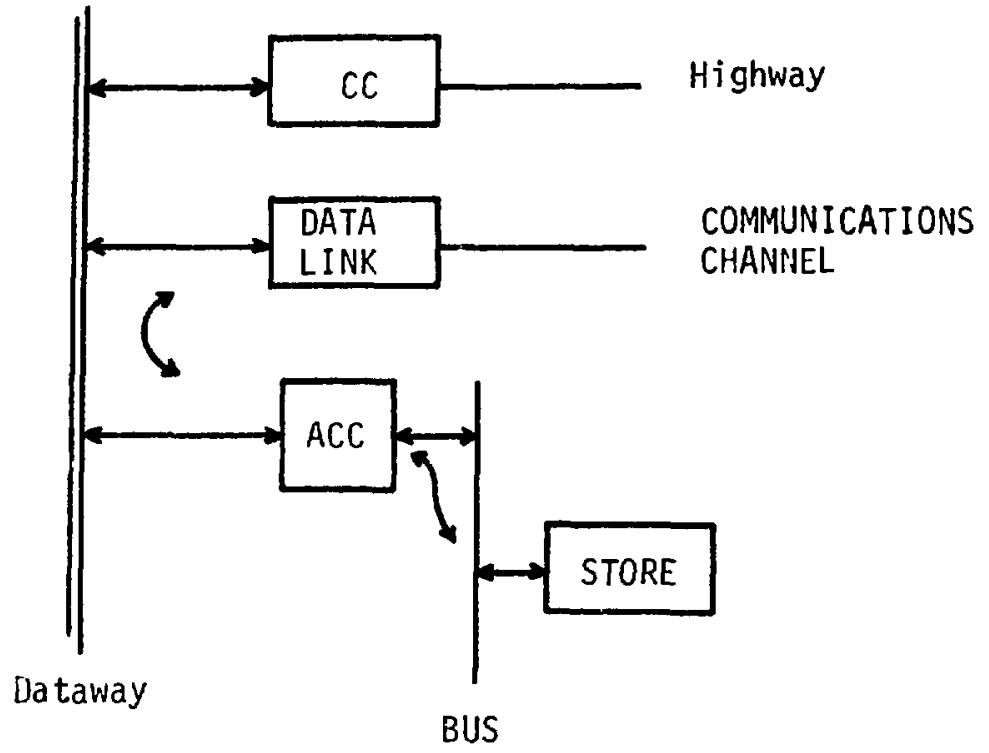

Another approach to the communication channel link exploits the processor (ACC) internal bus. The link obtains block data directly from the ACC store.

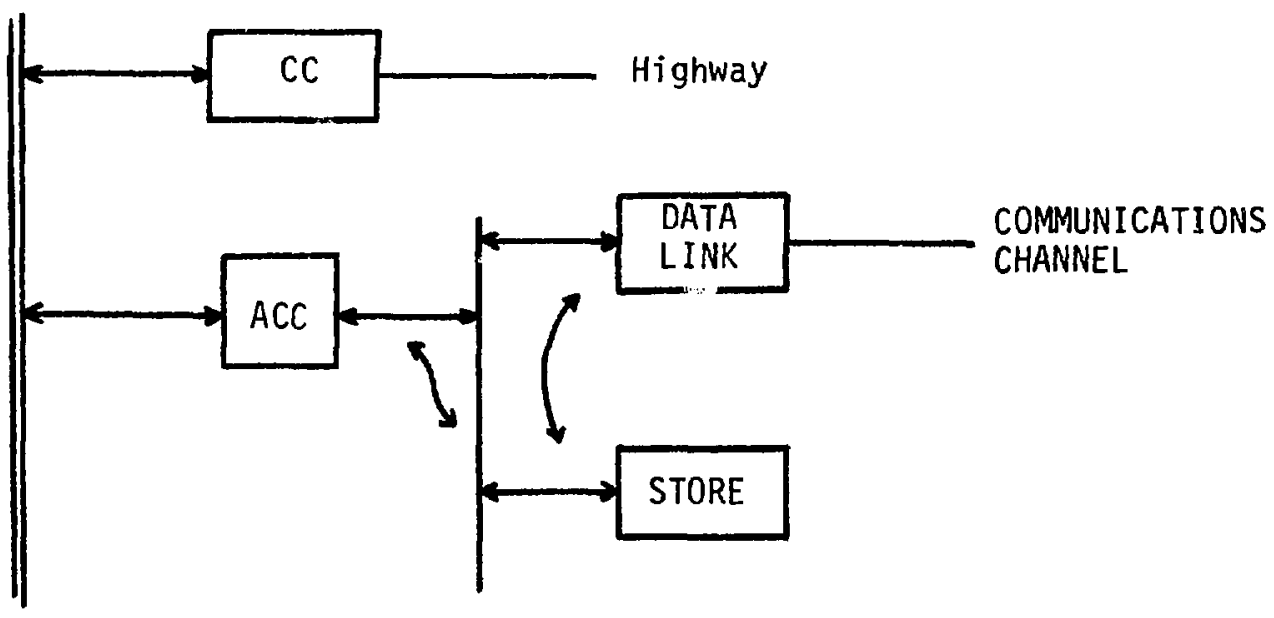

Da taway

BUS

- In the former method, the ACC must not only perform the in-crate processing task but must also provide information and protocol control to the data link module. The latter method eliminates one redundant path and allows $A C C$ and data link to function in parallel (if the data link were intelligent enough to DMA information from/to the store, and control itseif in addition). 
- The data link can, if microprogramed and given the appropriate po connection, generate serial highway protocol, SDLC/HDLC protocol, $n$ sbury Laboratory protocol, or any other communication channel protocol. Such a link implies a built-in processor similar to the ACC.

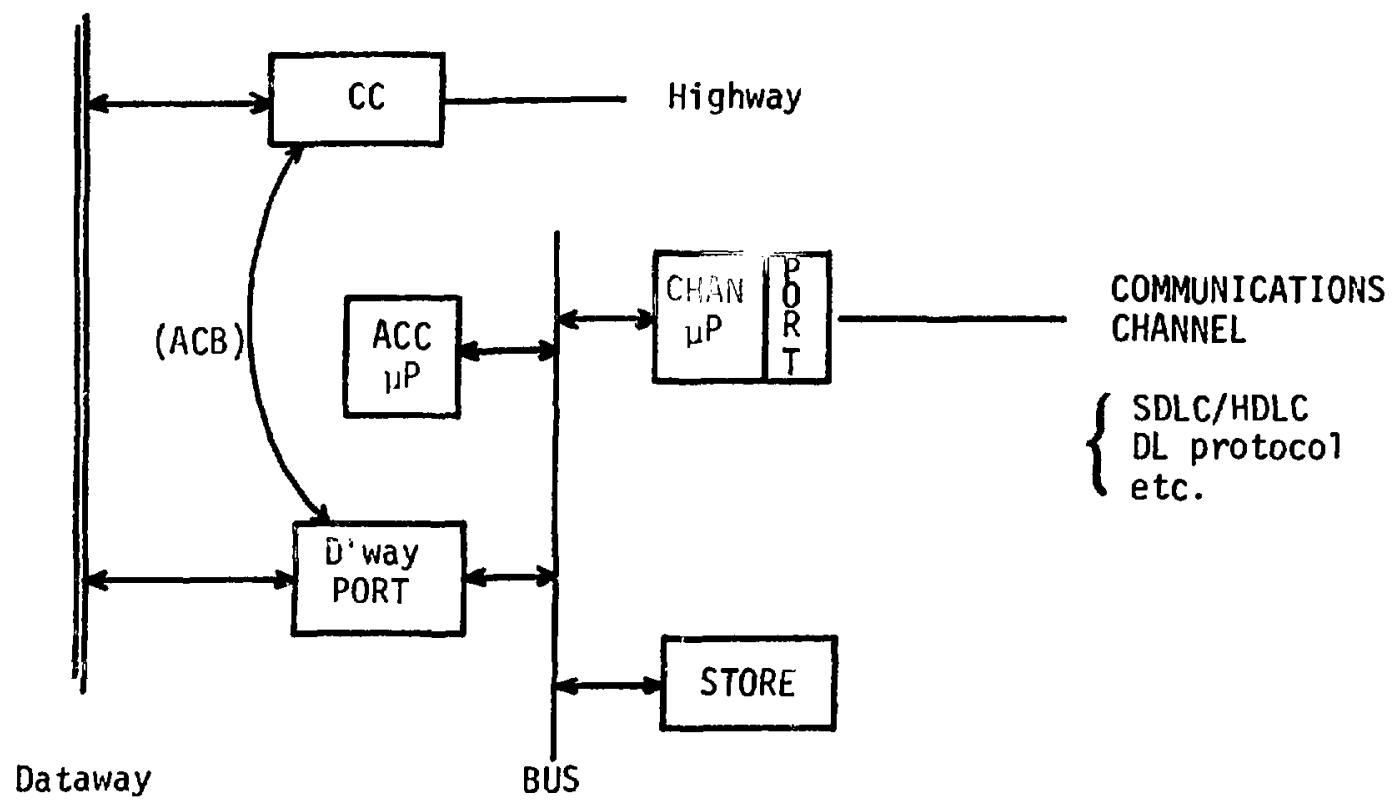


What About Crate-Crate Auxiliary Control?

One ACC in a crate cluster could have access to modules in two or more crates through the ACC internal bus.

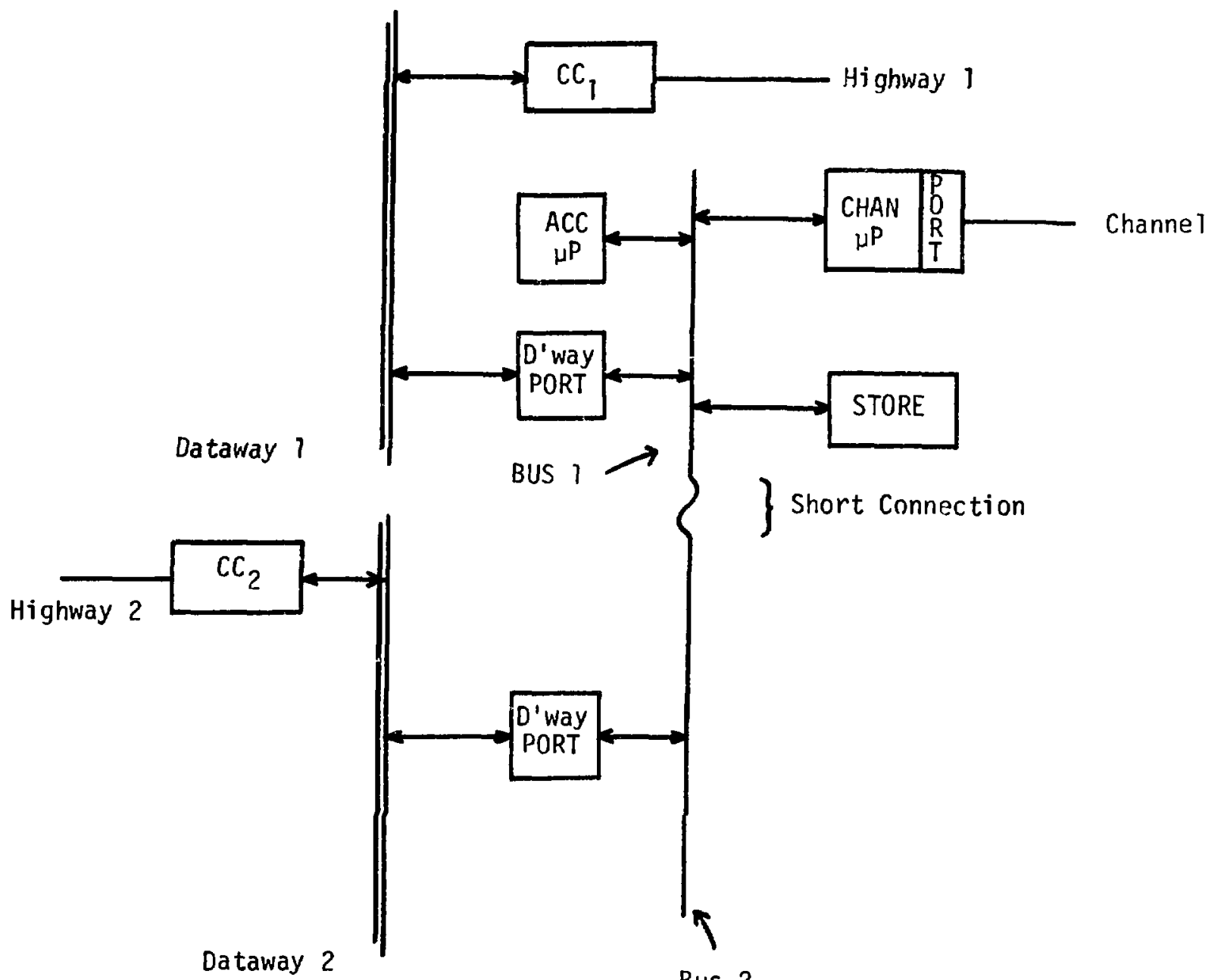

Bus 2

System considerations and microprocessor speed requirements could we11 limit the usefulness of this scheme. A complete ACC system in each crate may be desirable in any case.

Other Uses of the ACC

There is no fundamental reason why the ACC configuration presented in this note cannot be considered a bilateral one.

- Assume we have a system crate driving a CAMAC Highway in addition to providing both programmed and DMA interface to the system computer. Further assume that the crates connected to the Highway contain ACCs as described in this note. 
The CAMAC "channel" from each ACC is capable of sending along blockoriented data in contrast to the Highway, which is "single-action" oriented. How, then, do we convey this information to the system computer with the least overhead?

\section{Comm. Channel}

$\mathrm{SH}$

Other DL

HDLC/SDLC
Receiving Device

Special SD with block read/write protocol

An ACC configuration, interfaced to system crate

HDLC/SDL.C channel port (see note 4-76)

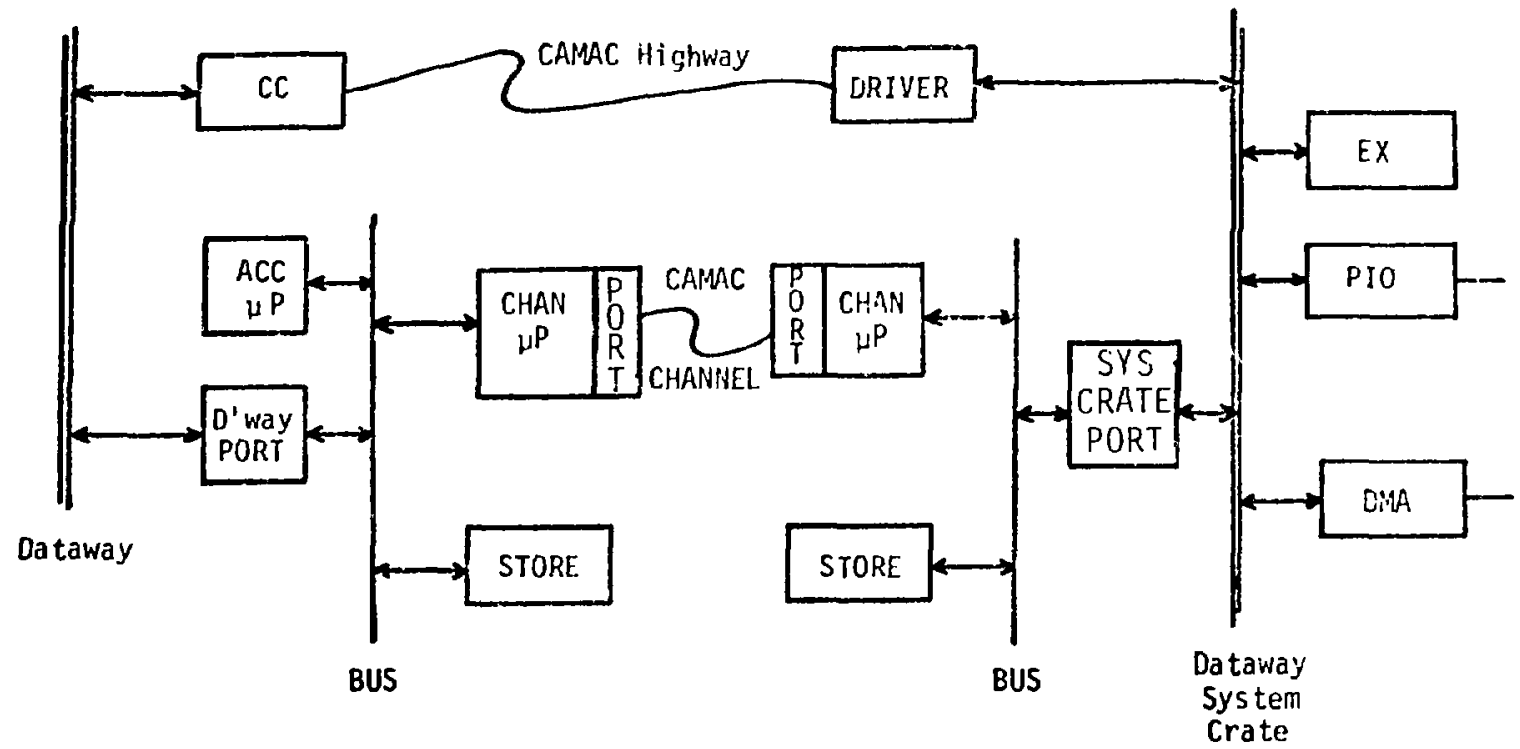

Further, the ACC with system crate interface could be used as a Serial Driver, provided the maximum performance of the Serial Highway was not desired. 


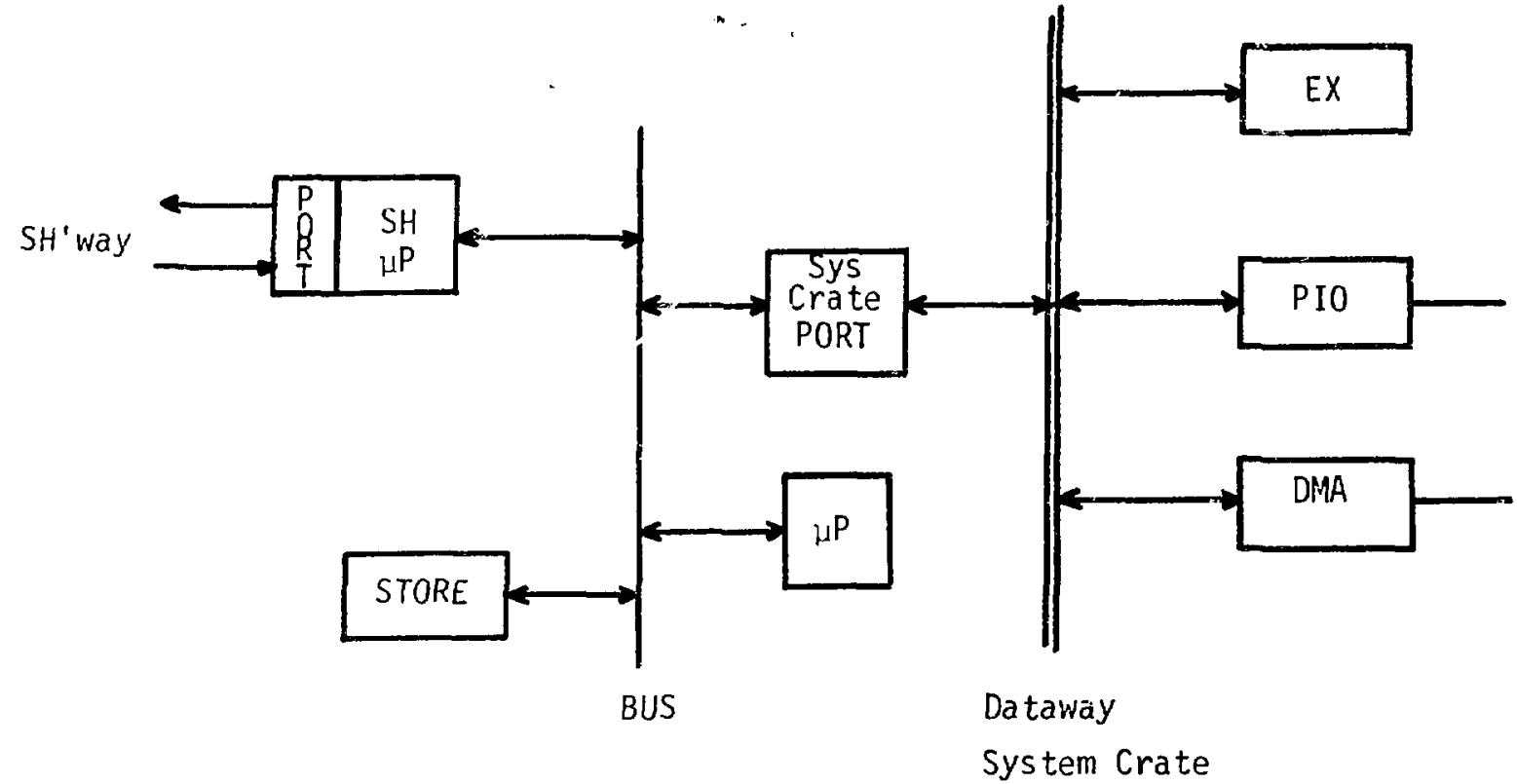

The use of the ACC in a system crate implies direct transfer cf information between the ACC and the system computer's memory, under control of the DMA controller.

It is conceivable that the DMI* controller could be replaced or significantly modified by such an ACC-oriented scheme!

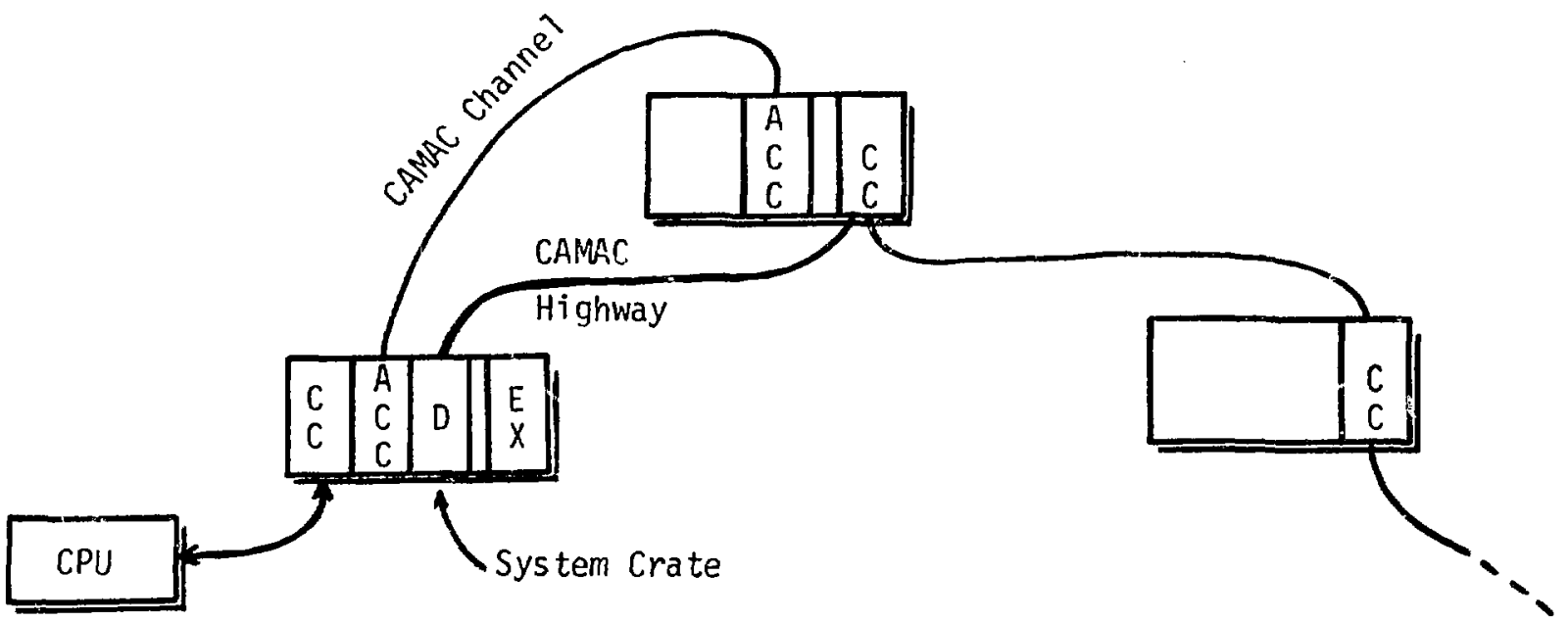

CAMAC ciannel information can be both data or macro instructions to ACC main processor imbedded within a message structure protocol (SH, SDLC/HDLC, DLDL, etc.).

*DMI - Direct Memory Increment. 


\section{CONVENTIONAL CAMAC SYSTEMS AND THE ACC ${ }^{\dagger}$}

Given the ACC components shown below as a minimal set of components to accomplish the auxiliary controller function, we must ask how the device fits into existing CAMAC structures and what it could do that is new.

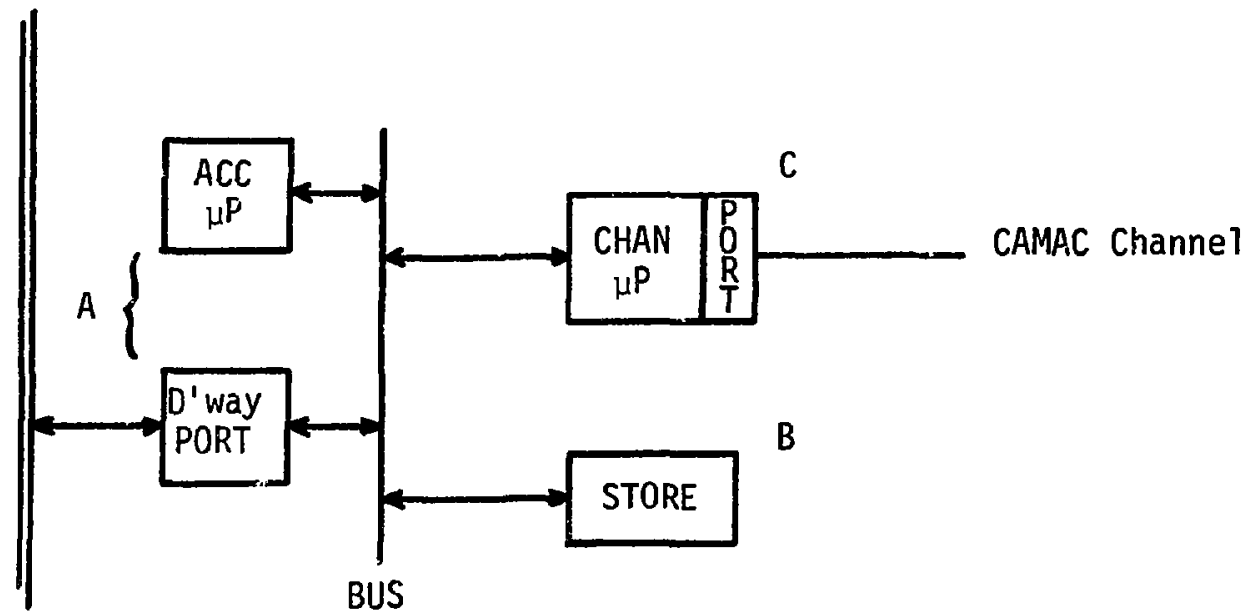

Dataway

The ACC processor/Dataway port (A), store (B), and channel processor/port (C) seem to be the lease one should assemble to create an ACC.

Perhaps the major point in favor of such an arrangement in a CAMAC crate with a conventional crate controller $(C C)$ is the mode of control that would be possible.

Cinventional CAMAC systems and CCs (see Fig. 1) communicate between CC and computer in a single-action mode; each CAMAC function to be executed must be passed to the $C C$ one at a time along the 1/0 bus or highway (DMA controllers do not, but they are rather special-purpose).

The ACC, with channel port, allows a CAMAC channel to communicate a message block between the crate and the central computer. The message blocks may cor. tain data to or from the computer, or it may contain a sequence of CAMAC instructions, or it may contain instructions to carry out some preprogrammed task. The data link scheme used at DL and LAMPF provides the same, except that one redundant path would be eliminated with ACC. The ACC, with or without the channel processor port, can serve to distribute tasks away from a central computer (much the same way as satellite minis do now). The difference is physical size and capability in an ACC without the channel port.

Capability

A 16-bit word length ACC processor seems reasonabie. Further, it would be an added feature to have available special CAMAC-oriented functions in microcode. One should not, however, try to build a computer or duplicate the general purpose computer instructions in a microcoded processor. * One proposal would be to provide a micronova TI 9900, or Ferranti Fl00L as the general purpose

TDaresbury Laboratory Note CSE 4-76, D. R. Machen.

*Microcoded processor or microprogramming facility, constructed with bipolar, bit-sliced chips. 
"provider" of instructions, and a companion facility, on the internal bus, that was capable of executing special and/or high-speed functions.

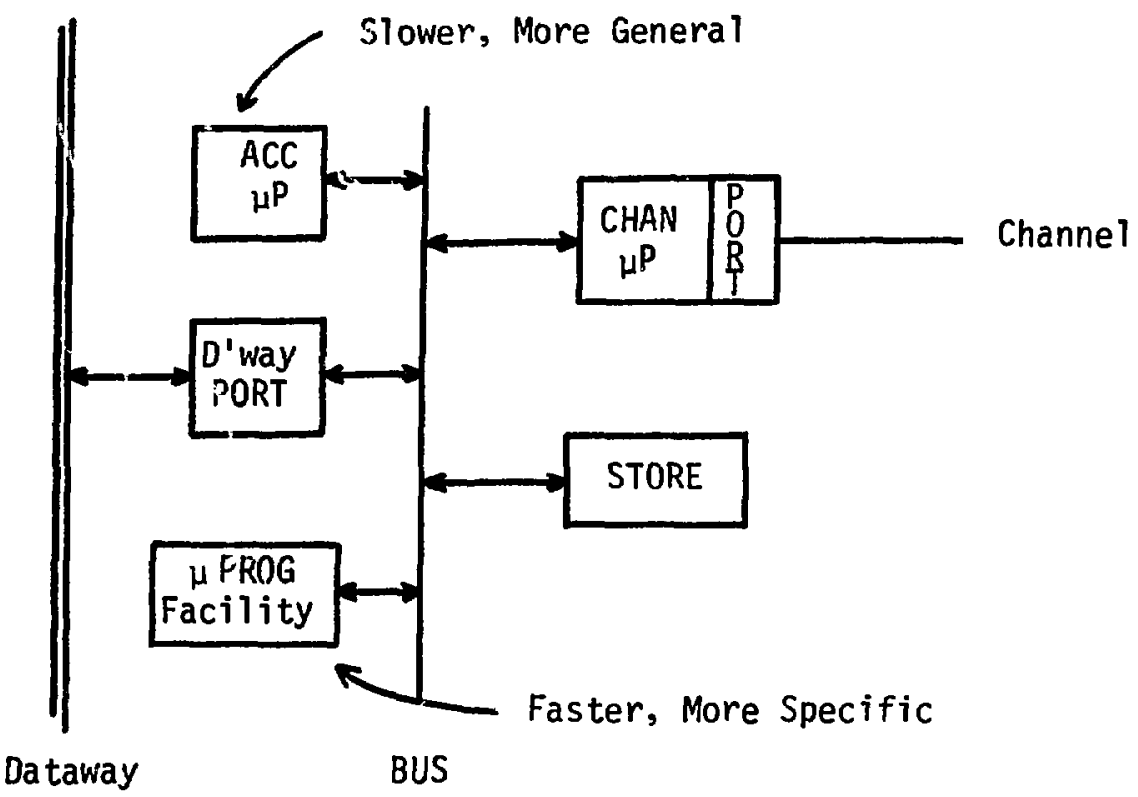

The microprogramming facility can be plugged in, or not, depending upon the task to be performed.

Figures $\mathrm{Al}$ to $\mathrm{A4}$ take each common CAMAC configuration with an ACC in an attempt to sort out the question of what does it buy us.

Addition of an ACC to (1) or (2) in Fig. Al does not seem to gain one much. Other configurations, however, have more appeal. Systems shown in Figs. A2 to A4 could provide a new degree of freedom to CAMAC instrumentation.

Figures $A 5$ and $A 6$ indicate the possible organization of the $A C C$ and system configuration, respectively. One must ask, what the messages to ACCs will contain, assuming that they are imbedded within a SDLC/HDLC message protocol? Shifting our thinking from Branch Highway and Serial Highway multicrate configurations to CAMAC channel configurations, may well allow CAMAC to be associated with some form of industry standard I/O bus, thus reducing the problems involved with computer-dependent drivers that exist today.

As I mentioned earlier in this note, the big advantage in moving to intelligence in the channel port and CAMAC controllers is the possibility it gives us to eliminate single-action commands and incorporate multiple-action commands. This is evident when we answer the question concerning the content of a CAMAC channet message.

1. Machine code for ACC (down-line loading ACC)

2. Single-action NAF

3. String of NAFs and data

4. String of data at single NAF

5. Higher level source to ACC; resident interpreter/compiler

6. Task execution

7. String of tasks 
On reflection, it is evident that No. 2, only, is common with present highway configurations. Further, by distributing the intelligence throughout a system we have moved the computer-to-dataway mapping away from the driver and into the crate, allowing a more sophisticated set of message possibilities along a more standard communicátions channel.

\section{Multi-crate Connections}

Multi-crate connections may be accomplished either through internal ACC bus extensions within crate clusters or through store and forward message handling at each ACC channel port. The latter has not been investigated in depth at this point, but seems to be one solution, based upon computer network practices. The lack of implicit intelligence in SCC led us to a unidirectional loop for the Serial Highway. With a message-oriented scheme, we should be able to do much better by using techniques developed for data communications networks. 
(1)

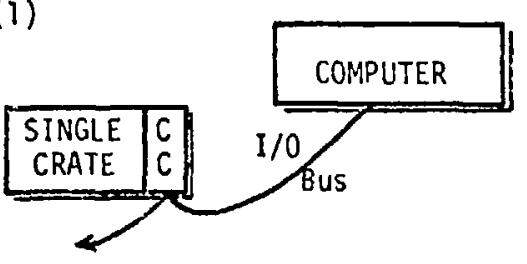

(3)

(4)

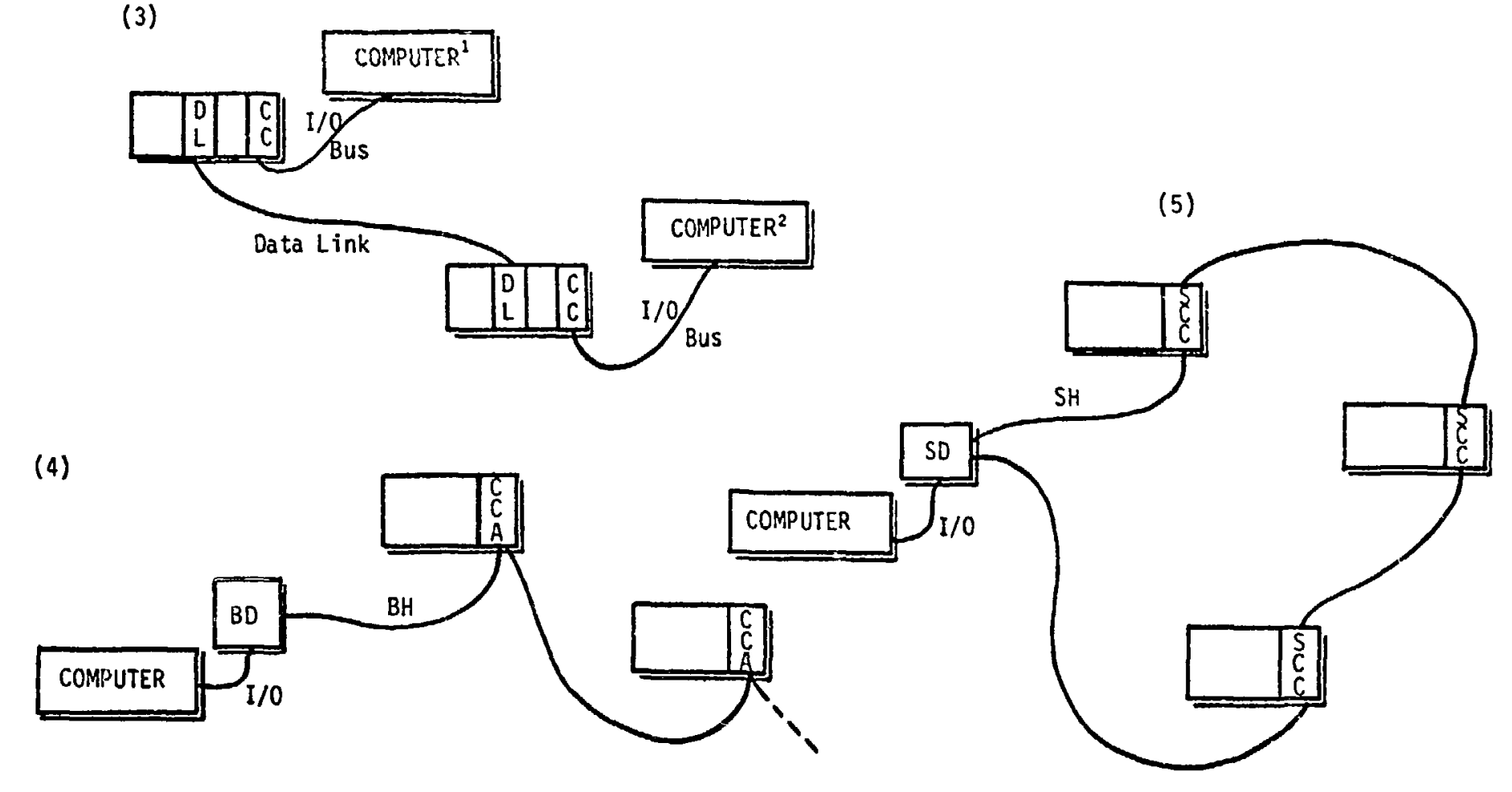

(2)

OR

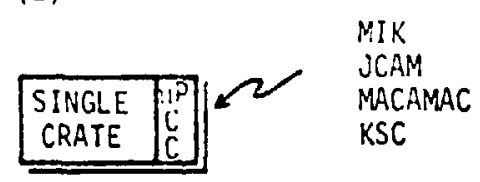

Fig. A-1. Typical CAMAC configurations. 


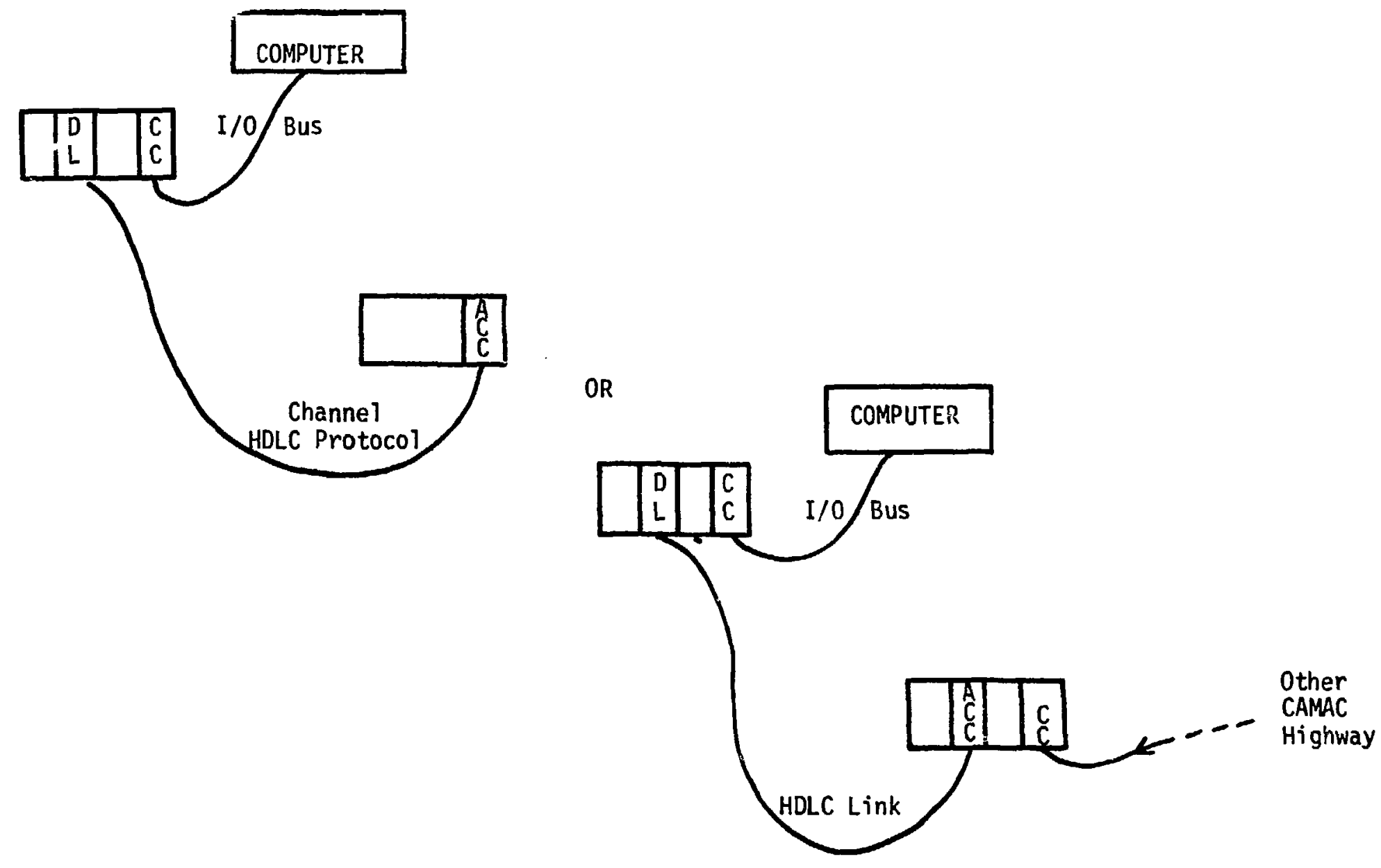

Fig. A-2. Data link coupled systems. 

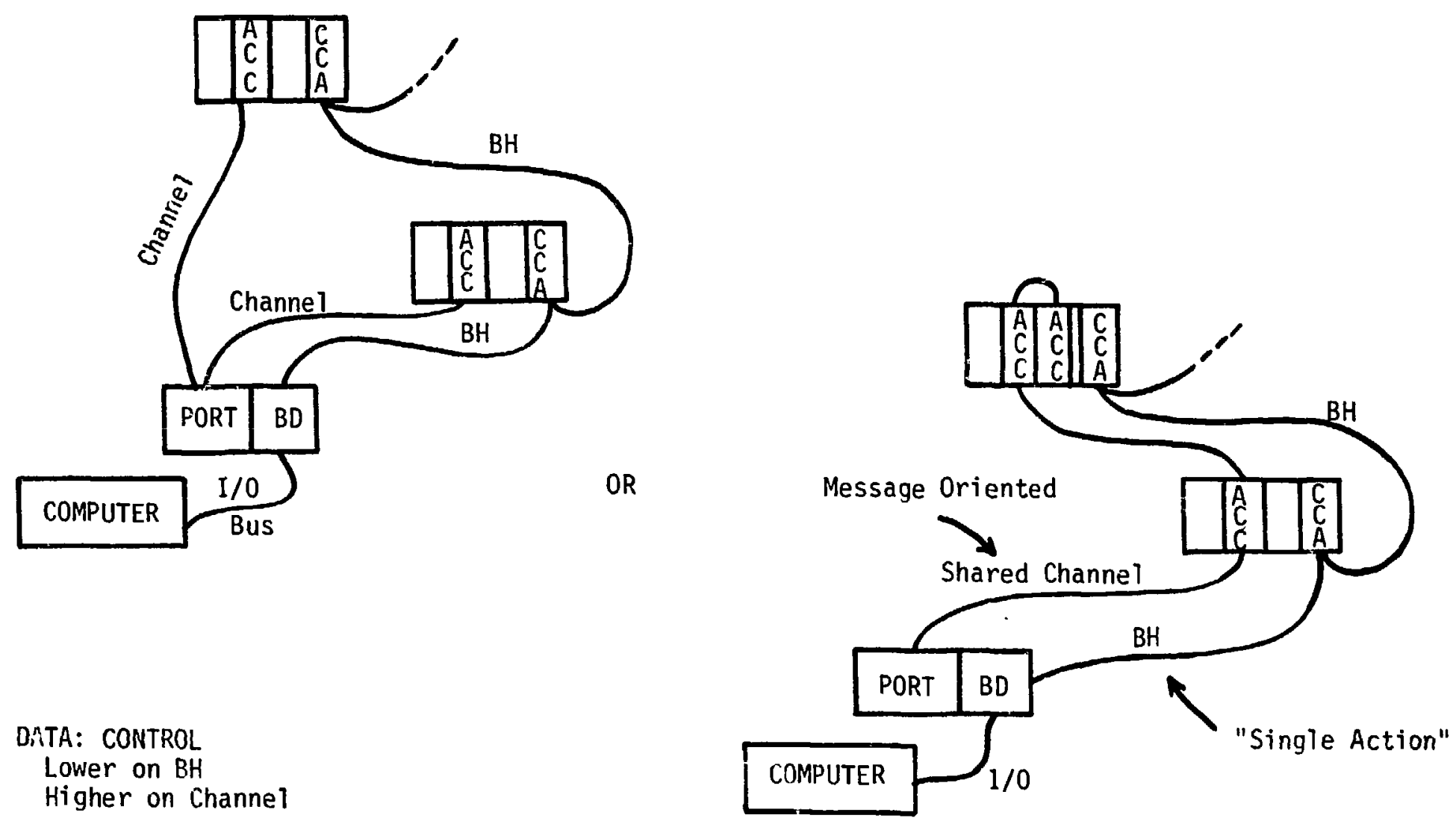

Fig. A-3. Branch highway systems. 


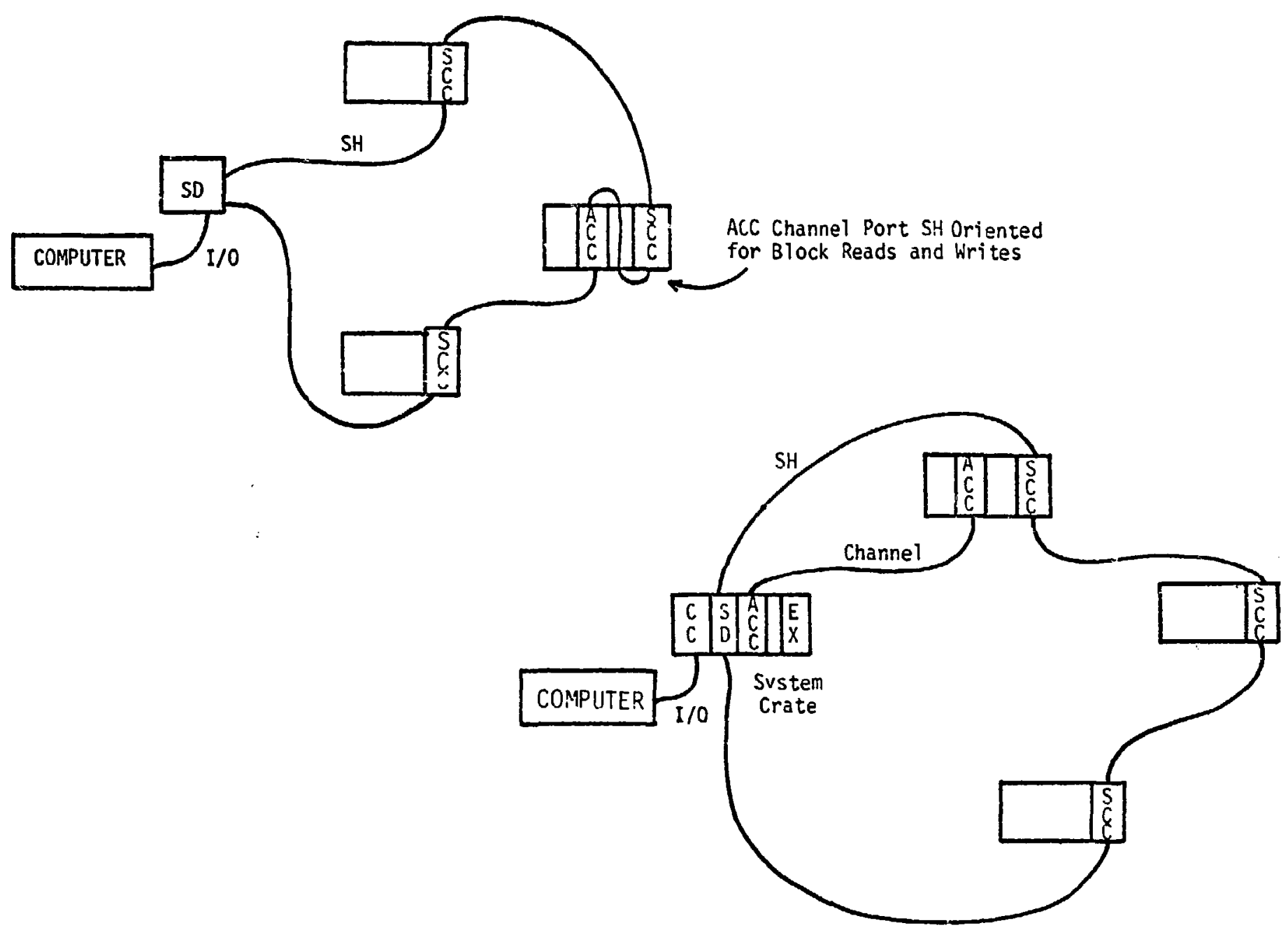

Fig. A-4. Serial highway systems. 


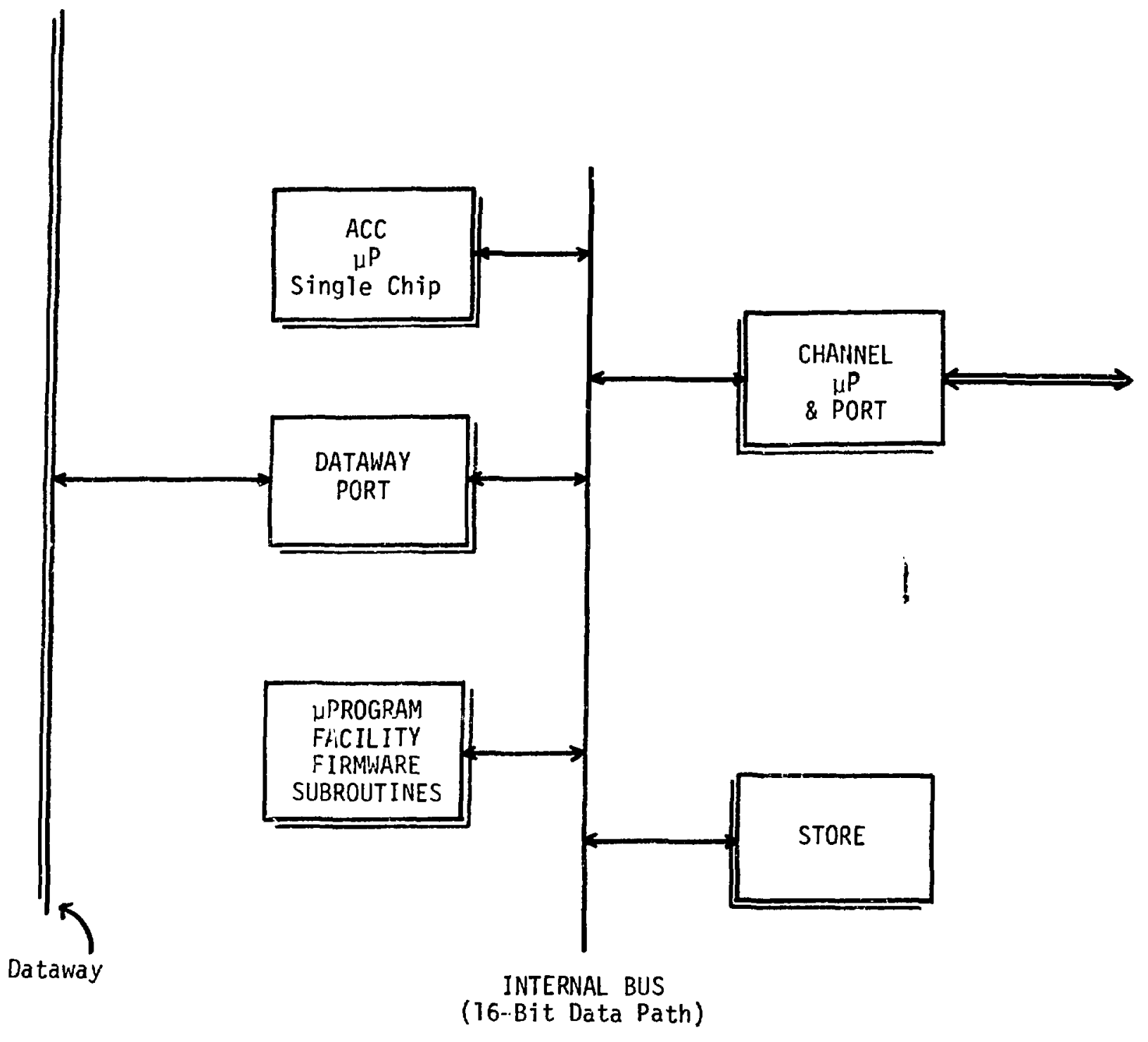

Fig. A-5. Recommended ACC configuration. 
SYSTEM CONTROLLER

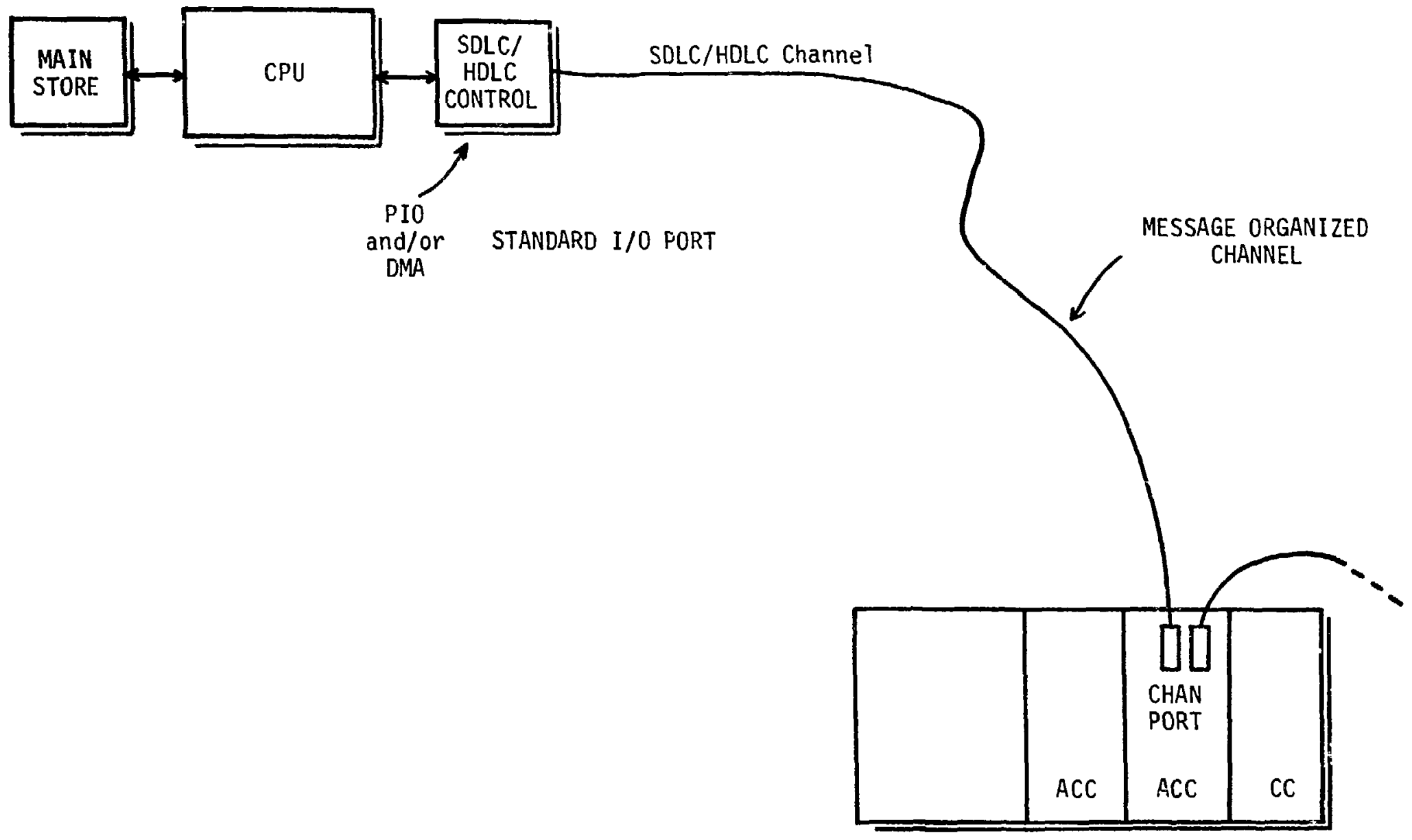

CAMAC CRATE

(Generalized Control)

Fig. A-6. CAMAC communications channel. 